\title{
Evaluation of optimal FVDs for inter-storey isolation systems based on surrogate performance models
}

\author{
Marco Donà ${ }^{1,2}$ D Enrico Bernardi ${ }^{1,2} \cdot$ Alberto Zonta $^{2} \cdot$ Ping Tan $^{1} \cdot$ Fulin Zhou $^{1}$
}

Received: 6 December 2020 / Accepted: 24 May 2021 / Published online: 8 June 2021

(c) The Author(s) 2021

\begin{abstract}
Inter-storey seismic isolation is increasingly gaining attention. One of the main related issues is the need to limit the relative displacement between substructure and superstructure, while maintaining a good seismic performance of the superstructure. As shown in some studies, fluid viscous dampers (FVDs) mounted in isolation systems are effective in reducing isolator deflection but can be harmful by amplifying inter-storey drifts and floor accelerations. Additionally, the effectiveness of FVDs for inter-storey applications was investigated only recently, and specific approaches for their optimisation and performance evaluation are missing. Therefore, this paper proposes a method for the optimal multiobjective design of FVDs, based on the definition of appropriate surrogate response models, which allows for rationally comparing the FVD effects for a wide range of dampers and structures. In particular, the optimal FVD parameters are provided in a dimensionless form, so that they can be predicted by design equations of general validity within the range of the structures analysed. This method is applied to a stock of regular structures with various vibration periods of superstructure, isolation and substructure, examining a linear and a non-linear isolation system and a set of natural records, in order to comprehensively assess the effects of FVDs and their non-linearity on the seismic performance of these structures. Finally, prediction models of optimal FVD parameters are provided based on the results obtained and are applied to three case studies as an example.
\end{abstract}

Keywords Inter-storey isolation - Supplemental viscous damping · Optimal design · Surrogate response models $\cdot$ Fluid viscous dampers (FVDs) $\cdot$ Linear and non-linear isolation systems

Marco Donà

marco.dona.1@unipd.it

1 Earthquake Engineering Research and Test Center, Guangzhou University, Guang Yuan Zhong Rd. 248, Guangzhou 510405, China

2 Department of Geosciences, University of Padova, Via Gradenigo 6, 35131 Padua, Italy 


\section{Introduction}

Seismic isolation between building storeys is becoming an increasingly attractive concept. First, it allows for greater freedom in the structural conception of skyscrapers and multi-purpose buildings, defining two independent structures, i.e., substructure and superstructure, which may have different forms, materials and uses (Zhang et al. 2017; Liu et al. 2018; Faiella and Mele 2020). This represents both an advantage for architectural design and a sustainable solution for densely populated areas (such as China), as it allows significant savings on land use, e.g., by realising residential buildings on top of commercial buildings. Additionally, in some cases, the base isolation of buildings encounters economic and technical issues that can prevent its application. For example, installing base isolation in existing buildings is generally complicated and certainly more expensive than applying isolation between storeys (often disruption-free). This technique can also be applied to add extra storeys on the top of existing buildings (with appropriate vertical capacity), without increasing the base shear forces, which represent an innovative retrofitting approach (Zhou 2001; Chey et al. 2013; Faiella et al. 2020). Furthermore, the isolation at the base becomes less effective than that between storeys for tall buildings, due to their low bending stiffness (Ziyaeifar et al. 1998).

Some examples of inter-storey isolation are as follows: the Shiodome Sumitomo Building (Tasaka et al. 2008) and the Iidabashi First Building (Zhang et al. 2017) in Tokyo, which are two multipurpose high-rise buildings consisting of a substructure and superstructure with different structural shapes; a building complex in Beijing (Zhou et al. 2004), where 50 base-isolated residential buildings (seven- or nine-storey RC frames) were built on top of a two-storey platform, covering a railway area of $\sim 3 \mathrm{~km}^{2}$; the 185 Berry St. building in the China Basin area of San Francisco (Dutta et al. 2009), which is the first example in the U.S. of an isolated building (two-storey steel structure) built on top of an existing building (three-storey RC structure); the nine-storey pre-cast RC building in the National Taiwan University campus (Loh et al. 2013), which has an inter-storey isolation system between the second and third floors, also equipped with viscous dampers.

This isolation strategy basically converts the mass of the isolated superstructure into a non-conventional tuned mass damper (Reggio and De Angelis 2015); therefore, the superstructure performs a dynamic control function in addition to the structural one. In general, it is possible to identify three behavioural categories-mass damping, intermediate isolation and base isolation - based on the mass ratio between the superstructure and substructure (Faiella and Mele 2019; Zhou et al. 2016; Tan et al. 2008).

One of the primary issues concerning inter-storey isolation is the need to reduce the P- $\Delta$ effects, by controlling the drift demand between the substructure and superstructure, while maintaining a good seismic performance of the superstructure (low floor accelerations and low inter-storey drifts). For this purpose, additional fluid-viscous dampers (FVDs) are effective in reducing the isolation drift demand and allow the design of a greater performing isolation system. Indeed, the isolation components can be designed for low activation forces, regardless of the actual expected seismic force, relying on the additional dissipation provided by these dampers.

In fact, dampers are often installed in base-isolated structures built near active faults, as the large displacement demand would otherwise require isolators of considerable size and cost (Koh et al. 1989; Makris 1997). However, studies on the supplemental damping in buildings with base isolation (e.g., Kelly 1999; Hall 1999; Alhan et al. 2004; Politopoulos 2008; Providakis 2008; Fathi et al. 2015) showed that high values of damping, when 
concentrated only at the isolation level, can be excessive and therefore harmful due to amplification of inter-storey drifts and floor accelerations. Both of these, on the other hand, can be limited by increasing the damping of the superstructure, which is effective in reducing the floor response spectra in correspondence with the second vibration mode of isolated structures (Ragni et al. 2020). Although non-linear dampers offer greater dissipation per sinusoidal cycle than linear ones (for the same maximum force and stroke) and limit the damper force transmission at high velocities (Tubaldi et al. 2015b), experiments carried out by Wolff et al. (2015) proved that these amplifications are smaller in case of linear dampers, especially with highly dissipative isolation devices.

The results of these studies are not always easy to compare, as they depend on specific assumptions, including FVD properties. Moreover, the effects of additional FVDs for interstorey applications were investigated only recently for a case study (Liu et al. 2018; Donà et al. 2019); therefore, further investigations are needed and should be addressed through a general assessment method, which allows comparing a wide range of linear and nonlinear dampers. Also, general studies on the optimisation of FVDs for such applications, addressed to their design, are missing. In fact, relevant studies in the literature mainly deal with the optimal arrangement of multiple isolation systems in buildings (Charmpis et al. 2012; Charmpis et al. 2015) and the optimal values of the isolator parameters (Reggio et al. 2015; Zhou et al. 2016). As for FVDs, many optimisation strategies are available for traditional applications in frames (e.g., see De Domenico et al. 2019), whereas only a few studies in the field of bridges deal with the optimisation of FVDs used together with isolators, focussing on linear dampers (Xie et al. 2017). Clearly, the use of FVDs in inter-storey isolation applications has some specificities, such as the damping, velocity and stroke values of the dampers-which are generally higher than in traditional applications-the concentration of energy dissipation between two storeys of the structure, and the interaction between the non-linear behaviour of the dampers and the isolation system.

Only recently, to assess the FVD effects in inter-storey applications, Liu et al. (2018) optimised the damper parameters (damping constant $c$ and linearity degree $\alpha$ ) individually for various seismic inputs, in the case of a seven-floor reinforced-concrete (RC) structure, with lead rubber bearings (LRBs) connecting the second and third floors. In addition to proving the effectiveness of FVDs, the authors showed that the optimal value of $\alpha$ is correlated with the structural seismic response (problem output) and strongly depends on the seismic input (a priori unknown). Therefore, a direct optimisation for each design ground motion is not suitable for design purposes, as $\alpha$ values cannot be averaged for determining the best FVD solution. In addition, the application of a multi-objective genetic algorithm to conduct a direct optimisation (as in Liu et al. 2018), even if based on the average of the maximum responses to a set of ground motions, would require the execution of a large number of non-linear time-history (TH) analyses, and therefore a high computational cost.

To overcome these issues, this paper proposes a more effective method for the optimisation and performance evaluation of FVDs for inter-storey applications, which falls within the multi-objective evolutionary design approach (De Domenico et al. 2019). This method is based on the definition of appropriate surrogate response models, calibrated on the maximum response (averaged between seismic inputs) of some significant performance parameters and their subsequent minimisation through multi-objective genetic algorithms. Such models, if conveniently expressed in a dimensionless form, allow obtaining results that can be compared between various case studies and predicted by design equations of general validity (within the range of structures analysed).

To systematically evaluate the FVD effects on the seismic performance of various structures, this method was parametrically applied to a stock of 48 lumped-mass models, 
representative of regular RC structures with various vibration periods of superstructure, isolation and substructure, analysing a wide range of damper forces for various $\alpha$ values between 0 and 1 , two types of isolation systems (linear with fuse behaviour and non-linear with LRB isolators) and a set of spectrum-compatible natural records. The conflicting objective functions (OFs) chosen for the optimal design are the minimisation of the drifts between the substructure and superstructure and the minimisation of the inter-storey drifts of the superstructure. In particular, these structural performances were normalised to the relevant ones in the case without FVD, to directly evaluate the effects of additional damping on the structural response. The multi-objective problem was solved through the NSGA-II genetic algorithm (Deb et al. 2002) and the optimisation results, both in terms of structural performances and optimal FVD parameters, were compared between various structural configurations and isolation systems to draw the relevant considerations. The maximum values of the superstructure floor accelerations and substructure inter-storey drifts were also evaluated, but as an output of the optimisation process rather than as OFs. The proposed method was then reapplied to optimise only linear FVDs $(\alpha=1)$, allowing performance comparisons between optimal linear and non-linear dampers. Finally, to provide useful design tools, prediction models for the optimal FVD parameters were defined and calibrated on the basis of the results obtained; these models were then applied to three case study structures, for example and validation purposes.

\section{Multi-objective optimisation method}

\subsection{Solution to the dynamic problem}

The equation of the dynamics of inter-storey isolated buildings with FVDs, with $t$ the time variable, is:

$$
\mathbf{M} \ddot{\mathbf{x}}(t)+\mathbf{C}_{\mathbf{S T}} \dot{\mathbf{x}}(t)+\mathbf{K}_{\mathbf{S T}} \mathbf{x}(t)+\mathbf{r}_{\mathbf{F V D}} \mathrm{F}_{\mathrm{FVD}}(t)+\mathbf{r}_{\mathbf{I S}} \mathrm{F}_{\mathrm{IS}}(t)=-\mathbf{M I}_{\mathrm{g}}(t)
$$

$\mathbf{x}(t)=\left[\begin{array}{llll}\mathrm{x}_{1}(t) & \mathrm{x}_{2}(t) & \ldots & \mathrm{x}_{\mathrm{n}}(t)\end{array}\right]^{\mathrm{T}}, \dot{\mathbf{x}}(t)$, and $\ddot{\mathbf{x}}(t)$ are respectively the floor displacements, velocities and accelerations relative to the building base, and $n$ is the number of degrees of freedom (DOF) examined; only translational DOF are taken into account by this modelling. $\ddot{\mathrm{u}}_{\mathrm{g}}(t)$ is the seismic acceleration, and $\mathbf{I}$ is the vector with the unitary rigid displacements of the floors, parallel to the seismic direction; in this work, $\mathbf{I}$ is the identity vector. $\mathbf{M}[n \mathbf{X}$ $n]$ is the global mass matrix and $\mathbf{C}_{\mathbf{S T}}$ and $\mathbf{K}_{\mathbf{S T}}$ are respectively the damping and stiffness matrices, given in Eq. 2, excluding the isolation system. $\mathbf{C}_{\mathbf{L}}$ and $\mathbf{K}_{\mathbf{L}}$ are respectively the damping and stiffness matrices for the lower structure (with $n_{L}$ DOF), and $\mathbf{C}_{\mathbf{U}}$ and $\mathbf{K}_{\mathbf{U}}$ are the same matrices for the upper structure (with $n_{U} \mathrm{DOF}$ ). In this study, the behaviour of the substructure and superstructure (and so $\mathbf{K}_{\mathbf{S T}}$ ) are modelled as linear, and all non-linearities are concentrated at the isolation level.

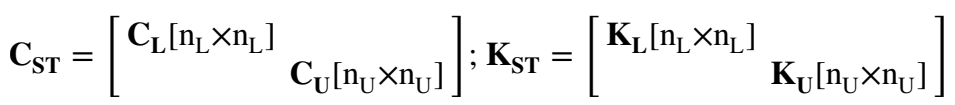

The structural equivalent viscous damping for such buildings is a 'non-classical damping' (Chen et al. 2017; Chen et al. 2019) and requires to be modelled differently for the two structural parts (Liu et al. 2018). The classic Rayleigh's model (Eq. 3), generally used for fixed-base structures, can also be assumed for the substructure; instead, a damping model 
proportional to stiffness (Eq. 4) is more appropriate for isolated structures, as discussed in Ryan et al. (2008) and Pant et al. (2013). In Eq. 3, $\mathbf{M}_{\mathbf{L}}\left[n_{L} \mathrm{x} n_{L}\right]$ is the mass matrix of the substructure and $\alpha_{L}$ and $\beta_{L}$ are the Rayleigh coefficients; the latter can be calculated by imposing a damping ratio $\zeta$ of $5 \%$ (usually assumed for RC buildings) to the modal frequencies $\omega_{i}$ and $\omega_{j}$, which determine the frequency range of interest of the lower structure. Regarding the superstructure damping model in Eq. 4, Pant et al. (2013) highlighted that such a model could provide excessive damping for higher modes in tall buildings (where they are significant). As discussed in Liu et al. (2018), this issue is also relevant for applications of inter-storey isolation, as the higher mode effects could be important for such buildings. Therefore, the proportionality coefficient $\beta_{U}$ should be calibrated by associating the desired $\zeta$ value (5\%) with a representative frequency $\omega_{k}$ for the higher modes to balance and limit this structural damping among the main higher modes (as shown in Liu et al. 2018). To assess the calibration of the matrix $\mathbf{C}_{\mathbf{S T}}$, the single modal damping ratios $\zeta_{i}$ can be calculated using Eq. 5, which is valid for classical damping but still allows sufficiently accurate estimates $-\boldsymbol{\phi}_{i}$ and $\omega_{i}$ represent the modal shape and pulsatance of the $i$-th mode, respectively. A good calibration should provide $\zeta_{i}$ of about $5 \%$ for the significant higher modes, and an almost zero damping for the first mode. As for the latter, the reason is that the dissipation contribution by deformation of the structural parts is negligible for this mode, with respect to that provided by the separation layer.

$$
\begin{gathered}
\mathbf{C}_{\mathbf{L}}=\alpha_{L} \mathbf{M}_{\mathbf{L}}+\beta_{L} \mathbf{K}_{\mathbf{L}} \rightarrow \alpha_{L}=\zeta \cdot \frac{2 \omega_{i} \omega_{j}}{\omega_{i}+\omega_{j}} ; \beta_{L}=\zeta \cdot \frac{2}{\omega_{i}+\omega_{j}} \\
\mathbf{C}_{\mathbf{U}}=\beta_{U} \mathbf{K}_{\mathbf{U}} \rightarrow \beta_{U}=\zeta \cdot \frac{2}{\omega_{k}} \\
\zeta_{i}=\frac{\boldsymbol{\Phi}_{i}^{T} \mathbf{C}_{\mathbf{S T}} \boldsymbol{\phi}_{i}}{2 \omega_{i} \boldsymbol{\phi}_{i}^{T} \mathbf{M} \boldsymbol{\phi}_{i}}
\end{gathered}
$$

The last two addenda of Eq. 1 describe the contribution of the isolation layer. In particular, $\mathrm{F}_{\mathrm{FVD}}(t)$ is the damper force, given by Eq. 6, which depends on the damping coefficient $c$ and the damping exponent $\alpha$. F IS $(t)$ is the restoring force developed by the isolation system, and it clearly depends on the isolation technology adopted. In general, $\mathrm{F}_{\mathrm{IS}}(t)$ is the sum of two contributions - one linear $\mathrm{F}_{\mathrm{IS}-l}(t)$, due to a linear or post-yielding stiffness $\left(k_{\mathrm{IS}}\right)$, and one hysteretic $\mathrm{F}_{\mathrm{IS}-h}(t)$. In recent years, the Bouc-Wen model (Ismail et al. 2009), shown in Eqs. 7 and 8, has often been used for modelling the hysteretic behaviour of isolators, thanks to its adaptability to a wide range of hysteretic laws through appropriate calibration of its parameters. In Eq. $7, k$ is the elastic stiffness, $r$ is the stiffness ratio $\left(=k_{\mathrm{IS}} / k\right)$ and $d_{y}$ is the yielding displacement of the isolation system; $z(t)$ is an extra state variable that defines the hysteretic loop shape through the dimensionless parameters $A, \beta, \gamma$ and $n$. Lastly, $\mathbf{r}_{\mathbf{F V D}}$ and $\mathbf{r}_{\text {IS }}$, provided in Eq. 9, are the vectors necessary to correctly place the contributions of damper and isolators in the motion equation.

$$
\begin{gathered}
\mathrm{F}_{\mathrm{FVD}}(t)=c|\dot{x}|^{\alpha} \operatorname{sgn}(\dot{x}) \\
\mathrm{F}_{\mathrm{IS}}(x(t), \dot{x}(t))=\mathrm{F}_{\mathrm{IS}-l}(t)+\mathrm{F}_{\mathrm{IS}-h}(t)=k_{\mathrm{IS}} x(t)+\left(k-k_{\mathrm{IS}}\right) d_{y} z(t)=r k x(t)+(1-r) k d_{y} z(t)
\end{gathered}
$$




$$
\begin{aligned}
& \dot{z}(t)=\left(A \dot{x}(t)-\beta|\dot{x}(t)| \cdot z(t) \cdot|z(t)|^{n-1}-\gamma \dot{x}(t)|z(t)|^{n}\right) / d_{y}
\end{aligned}
$$

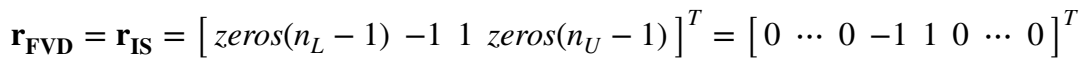

According to Eq. 10, the building's total restoring force $\mathbf{F}_{\mathbf{R}}(t)$ can be divided into two addends: one linear, provided by the elastic stiffness of the structure $\left(\mathbf{K}_{\mathbf{S T}}\right)$ and the linear component of the isolation force $\mathrm{F}_{\mathrm{IS}-l}(t)$, and one non-linear, due to $\mathrm{F}_{\mathrm{IS}-h}(t)$. Using Eq. 10 in Eq. 1 and solving for $\ddot{\mathbf{x}}(t)$ results in Eqs. 12 and 13 .

$$
\begin{aligned}
& \mathbf{F}_{\mathbf{R}}(t)=\mathbf{K}_{\mathbf{S T}} \mathbf{x}(t)+\mathbf{r}_{\mathbf{I S}} \mathrm{F}_{\mathrm{IS}}(t)=\left(\mathbf{K}_{\mathbf{S T}}+\mathbf{r}_{\mathbf{I S}} k_{\mathrm{IS}}\right) \mathbf{x}(t)+\mathbf{r}_{\mathbf{I S}} \mathrm{F}_{\mathrm{IS}-h}(t)=\mathbf{K} \mathbf{x}(t)+\mathbf{r}_{\mathbf{I S}}(1-r) k d_{y} z(t) \\
& \left.\mathbf{K}=\left[\begin{array}{ccc}
k_{1 L}+k_{2 L} & -k_{2 L} & \\
-k_{2 L} & \ddots & -k_{n L} \\
& -k_{n L} & k_{n L}+k_{I S}
\end{array}\right]\left[\begin{array}{ccc}
0 & & 0 \\
& \ddots k_{I S} & \\
0 & -k_{I S} \\
0 & \ddots & 0
\end{array}\right]\left[\begin{array}{ccc}
k_{I S}+k_{1 U} & -k_{1 U} & \\
-k_{1 U} & \ddots & -k_{n U} \\
& -k_{n U} & k_{n U}
\end{array}\right]\right] \\
& \mathbf{M} \ddot{\mathbf{x}}(t)+\mathbf{C}_{\mathbf{S T}} \dot{\mathbf{x}}(t)+\mathbf{K} \mathbf{x}(t)+\mathbf{r}_{\mathbf{I S}}(1-r) k d_{y} z(t)+\mathbf{r}_{\mathbf{F V D}} \mathrm{F}_{\mathrm{VD}}(t)=-\mathbf{M I} \ddot{u}_{\mathrm{g}}(t) \\
& \ddot{\mathbf{x}}(t)=-\mathbf{M}^{-1}\left(\mathbf{C}_{\mathbf{S T}} \dot{\mathbf{x}}(t)+\mathbf{K x}(t)+\mathbf{r}_{\mathbf{I S}}(1-r) k d_{y} z(t)+\mathbf{r}_{\mathbf{F V D}} \mathrm{F}_{F V D}(t)\right)-\mathbf{I}_{\mathrm{g}}(t)
\end{aligned}
$$

Finally, the governing equation can be properly rewritten, as in Eq. 14, by means of the state space vector $\mathbf{q}(t)=\left[\mathbf{x}^{\mathbf{T}}(t) \dot{\mathbf{x}}^{\mathbf{T}}(t) z(t)\right]^{\mathbf{T}}$, so that it can be solved as a first order differential equation. The vector $\mathbf{f}(\mathbf{q}(t))$ is obtained by deriving $\mathbf{q}(t)$, and then using Eqs. 8 and 13; $\mathbf{B}$ is the position vector for the seismic input.

$$
\begin{gathered}
\dot{\mathbf{q}}(t)=\left[\dot{\mathbf{x}}^{\mathbf{T}}(t) \ddot{\mathbf{x}}^{\mathbf{T}}(t) \dot{z}(t)\right]^{\mathbf{T}}=\mathbf{f}(\mathbf{q}(t))-\mathbf{B} \ddot{\mathbf{u}}_{\mathrm{g}}(t) \\
\mathbf{f}(\mathbf{q}(t))=\left[\begin{array}{c}
\dot{\mathbf{x}}^{\mathbf{T}}(t) \\
-\mathbf{M}^{-1}\left(\mathbf{C}_{\mathbf{S T}} \dot{\mathbf{x}}(t)+\mathbf{K} \mathbf{x}(t)+\mathbf{r}_{\mathbf{I S}}(1-r) k d_{y} z(t)+\mathbf{r}_{\mathbf{F V D}} \mathrm{F}_{F V D}(t)\right) \\
\left(A \dot{x}-\beta|\dot{x}| z|z|^{n-1}-\gamma \dot{x}|z|^{n}\right) / d_{y}
\end{array}\right] \\
\mathbf{B}=\left[\begin{array}{lll}
\mathbf{O} & \mathbf{I} & 0
\end{array}\right]^{\mathrm{T}} .
\end{gathered}
$$

\subsection{Response surfaces as a function of dimensionless FVD parameters}

Following the procedure above, parametric non-linear TH analyses can be performed for each structure and set of accelerograms assumed, by varying the FVD parameters $(c$ and $\alpha$ ) within the relevant range of interest. To examine an appropriate and sufficiently wide range of additional FVDs, the first-mode damping ratios $\left(\zeta_{F V D, l}\right)$ provided by linear FVDs $(\alpha=1)$, defined as in Eq. 17, can be initially set, from which the associated linear damping coefficients $c_{l}$ can be easily derived. $\mathbf{C}_{F V D, l}$ is the damping matrix that contains only the damper contribution $\left(c_{l}\right)$ 
and $\phi_{1}, \omega_{1}, M_{1}$ and $c_{c r l}$ are, respectively, mode shape, angular frequency, mass and critical damping of the first mode; $\psi_{1, n L}$ and $\psi_{1, n L+1}$ are the components of $\boldsymbol{\phi}_{1}$ at the isolation level.

$$
\zeta_{F V D, l}=\frac{\boldsymbol{\phi}_{1}^{T} \mathbf{C}_{F V D, l} \boldsymbol{\phi}_{1}}{2 \omega_{1} \boldsymbol{\phi}_{1}^{T} \mathbf{M} \boldsymbol{\phi}_{1}}=\frac{c_{l}\left(\psi_{1, n L}-\psi_{1, n L+1}\right)^{2}}{2 \omega_{1} M_{1}}=\frac{c_{l}\left(\psi_{1, n L}-\psi_{1, n L+1}\right)^{2}}{c_{c r 1}}
$$

Then, to define non-linear FVDs $(\alpha<1)$ comparable with linear ones in terms of maximum force, Eq. 18 can be used to calculate the relevant damping coefficients $c_{n l}$. In Eq. 18, $F_{F V D, l}$ and $v_{F V D, l}$ are respectively the maximum force and velocity of the reference linear FVD obtained from the $\mathrm{TH}$ analysis.

$$
c_{n l}=\frac{F_{F V D, l}}{\left(v_{F V D, l}\right)^{\alpha}}
$$

Therefore, from a chosen value of $\zeta_{F V D, l}$, a linear FVD can be determined by Eq. 17 ; then, from the maximum seismic response of this damper, comparable non-linear FVDs can be defined through Eq. 18 by setting various $\alpha$ values $(<1)$. Actually, with the same structure and seismic input, providing damping with different linearity degrees $(\alpha)$ results in different velocity responses, and thereby in different damping forces. However, this variation is limited, and the proposed procedure solely aims to define an appropriate range of non-linear FVDs on a rational basis. The $\zeta_{F V D, l}$ and $\alpha$ values examined in this study are reported in Table 1 and correspond to 120 case studies, in addition to the reference case without damper (i.e., $\zeta_{F V D, l}=0$ ).

The maximum values of a structural performance parameter, associated with the various accelerograms, can be averaged per storey if the number of accelerograms is code compliant. Thus, the peak response surface of that parameter can be obtained by plotting and interpolating its averaged maximum response versus the FVD parameters, over the entire range of analysed FVDs.

The parameters generally used to define the FVD are $c\left[\mathrm{~N}(\mathrm{~s} / \mathrm{m})^{\alpha}\right]$ and $\alpha[-]$. However, in place of $c$, a more convenient parameter, $r_{F}[-]$, is introduced in Eq. 19 for the purposes of this study; $r_{F}$ returns the maximum analysis value of the FVD force $\left(F_{F V D}\right)$, normalised to the maximum damper force obtained with $\zeta_{F V D, l}=1$ and $\alpha=1$, that is $F_{c r l}$, which is critical for the first vibration mode. For a given structure, each value of $\zeta_{F V D, l}$ ideally corresponds to a single value of $r_{F}$ for all the $\alpha$ examined. Actually, due to the simplification in Eq. 18, $r_{F}$ is very similar but not identical. The ratio $r_{F}$, in addition to providing more regular and appropriate response surfaces for subsequent FVD optimisation, is dimensionless, and therefore allows to effectively compare the peak responses of various structures as a function of dimensionless variables only.

$$
r_{F}=\frac{F_{F V D}}{F_{c r 1}}=\frac{F_{F V D}}{F_{F V D, l}\left(\zeta_{F V D, l}=1 ; \alpha=1\right)}
$$

Table 1 Examined values of $\zeta_{F V D, l}$ (first-mode damping ratio provided by linear FVDs) and $\alpha$

\begin{tabular}{ll}
\hline$\zeta_{F V D, l}(-)$ & $0,0.05,0.1,0.15,0.2,0.3,0.4,0.5,0.6,0.7,0.8,0.9$, \\
$\alpha(-)$ & 1
\end{tabular}




\subsection{Dimensionless surrogate response models}

For each performance parameter, an appropriate analytical model can be calibrated on the related response surface. These models, known as surrogate response models, are useful for overcoming the direct optimisation issues discussed in the Introduction. Moreover, they allow a graphical representation of the structural performances, which is very useful for a full understanding of the effects of the analysed variables that is impossible with direct optimisation.

The performance and generality of these models depend on the structural response (and therefore on the type of structure and seismic action), on the definition of the optimisation variables, and obviously on the analytical law assumed. In the subsequent parametric study, the complete fourth degree polynomial in $\alpha$ and $r_{F}$ was used as the analytical model, as it proved to be the most suitable in terms of fit and simplicity, among those evaluated.

Then, for a more effective assessment of the FVD effects, the structural response can be normalised to the maximum response in the case without the damper $\left(\zeta_{F V D, L}=0\right)$, thus deriving surrogate response models that are completely dimensionless (together with the variables $r_{F}$ and $\alpha$ ). These dimensionless models are also particularly suitable for parametric analyses that examine various structures and/or seismic inputs, allowing the optimisation results to be properly compared between various case studies, both in terms of FVD parameters and structural performance. Furthermore, surrogate models can be extended to include additional variables to those of the FVD, such as structure or earthquake parameters. However, the convenience of global models with many variables, compared to the many models dependent only on $r_{F}$ and $\alpha$, specific to a type of structure and/or seismic input, should be evaluated on a case-by-case basis, according to the purposes of the study. In fact, the greater the number of variables of the models, the lower is their prediction capability.

Furthermore, to effectively use the optimisation results for design purposes, in this phase, it is necessary to define a prediction model of $c$ as a function of $r_{F}$ and $\alpha$, which will allow to obtain the optimal $c$ values once the optimal $r_{F}-\alpha$ solutions have been calculated, because $c$ is not directly related to $r_{F}$. Then, to represent the results more effectively and compare them between various case studies, $c$ can be normalised as in Eq. 20, derived from Eq. 17, replacing $c_{l}$ with $c$.

$$
c^{*}=\frac{c}{c_{c r 1}}\left(\psi_{1, n L}-\psi_{1, n L+1}\right)^{2} .
$$

\subsection{Optimisation of FVDs and prediction of optimal results}

To determine the best FVD parameter sets $\left(r_{F}-\alpha\right.$ and then $\left.c-\alpha\right)$, some design aims must be defined. As already stated, dampers in inter-storey applications aim to reduce P- $\Delta$ effects and could be harmful to the isolated structure, increasing internal forces. Therefore, this optimal design is addressed to the resolution of conflicting objective functions (OFs), corresponding to the minimisation of the following surrogate response models:

- Drift between the lower and upper structures $\left(O F_{1}\right)$.

- Maximum inter-storey drift of the upper structure $\left(\mathrm{OF}_{2}\right)$. 
Superstructure floor accelerations can also be amplified by the additional damping, and therefore should be assessed, particularly for seismic protection of the structural content. These accelerations, as well as the performance of the substructure, were conveniently evaluated as an output of the optimisation problem rather than as variables of the optimisation criteria. In particular, to avoid significant damage to structural contents, a generally accepted limit of floor acceleration is about $0.3 \mathrm{~g}$ (Charmpis et al. 2012). Lower values may be required for special and fragile contents, for which, however, specific isolation systems exist (Donà et al. 2017).

Additionally, technical effectiveness is generally not the only decision-making aspect for the choice of the dampers, technical issues and costs associated with their implementation are also significant. Indeed, in seismic retrofit applications, with dampers distributed along the building height, the optimisation of the intervention generally aims at minimising specific cost functions; these may contain various cost components such as (Pollini et al. 2017) cost of preparing the structure for the installation of the dampers, manufacturing cost of the dampers (depending on their peak stroke and peak force), and additional costs for the use of dampers of different sizes due to the greater number of acceptance tests required by the standards. Often, especially when using the same type of device, this optimisation is performed by directly minimising the sum of the maximum damper forces (Altieri et al. 2018) or, for simplicity, the sum of the viscous damping constants (Tubaldi et al. 2015a). Regarding the inter-storey applications considered in this study, the peak stroke and force of the FVDs are both important to define the cost function. Moreover, a cost-effectiveness analysis of optimal solutions would also require the evaluation of possible savings on isolators and the substructure, due to the reduction of the isolation drift and the P- $\Delta$ effects on the substructure. However, since these latter factors significantly depend on a specific case study, these analyses can be more conveniently performed a posteriori for the optimal solutions obtained (out of the scope of this paper), in order not to reduce the generality of the study.

The solution of a multi-objective optimisation problem requires the determination of the Pareto front, i.e., a series of possible optimal solutions in which, generally, there is not hierarchy of preference. To date, several multi-objective optimisation algorithms allow to calculate the Pareto front, such as the evolutionary algorithms (Coello Coello et al. 2007). Among the latter, the fast and élitist non-dominated sorting genetic algorithm NSGA-II (Deb et al. 2002) was chosen for this study. In fact, the use of surrogate response models makes the choice of the algorithm less important, as the processing time for minimising these models is generally low; therefore, a different choice is possible and this would not affect the validity of this study.

Finally, with the aim of providing useful design tools, prediction models of the optimal FVD parameters can be defined and calibrated based on the results obtained.

\section{Application of the optimisation method to a parametric study}

\subsection{Structural models}

The optimisation method was parametrically applied to a stock of multi-degrees of freedom (MDOF) models, representing a wide range of regular RC frame structures, from low to high-rise. These MDOF models were defined on the basis of 3-DOF reference systems (Wang et al. 2011), characterised by the vibration period $(T)$ or angular frequency $(\omega)$ of 
the isolated superstructure (which includes the mass $m_{I S}$ of the isolation slab) $-T_{I S}$ or $\omega_{I S}$, superstructure $-T_{U}$ or $\omega_{U}$, and substructure $-T_{L}$ or $\omega_{L}$. The values of $\omega_{U}$ and $\omega_{L}$ were conveniently derived from the ratios $r \omega_{U}=\omega_{U} / \omega_{I S}$, for the superstructure and $r \omega_{L}=\omega_{L} / \omega_{I S}$ for the substructure. The analysed values of $T_{I S}$ were two, three and four seconds and the ratios $r \omega_{U}$ and $r \omega_{L}$ ranged from three to six.

To define the mass and stiffness matrices of the MDOF models, first the 3-DOF systems were calibrated on regular RC frames with inter-storey height $\left(h_{i}\right)$ of three meters and storey mass $\left(m_{i}\right)$ of 900 tons (corresponding, e.g., to a floor of $900 \mathrm{~m}^{2}$ with afferent mass of $1000 \mathrm{~kg} / \mathrm{m}^{2}$ ). In particular, the simplified relation between the building's height and principal vibration period provided in Eurocode 8 (EC8) (CEN 2004) for regular RC frames (see Fig. 1) was used to calculate the heights of superstructure $H_{U}$ and substructure $H_{L}$. Then, the associated masses $M_{U}$ and $M_{L}$ were derived on the basis of $h_{i}$ and $m_{i}$ and the associated stiffnesses $K_{U}$ and $K_{L}$, as well as the isolation stiffness $k_{I S}$, were calculated on the basis of the periods (or frequencies) of the 3-DOF system, initially assumed.

Subsequently, after defining masses and stiffnesses of the 3-DOF systems, the DOF representing the superstructure was replaced by an equivalent MDOF system with uniform masses $\left(m_{i}\right)$ and stiffnesses $\left(k_{i, U}\right)$. The aim is to properly assess the amplification effects of the superstructure modes due to the additional damping (in terms of inter-storey drifts and floor accelerations), which is not possible by modelling the superstructure as a single DOF.

Figure 1 summarises the parametric analysis data and the procedure used to define the MDOF models. The total analysed structures are 48 (for each FVD defined in Table 1), corresponding to the combination of three isolation periods $\left(T_{I S}\right)$ with four frequency ratios both for the superstructure $\left(r \omega_{U}\right)$ and substructure $\left(r \omega_{L}\right)$.

The minimum values of $r \omega_{U}$ and $r \omega_{L}$ (equal to three), consistent with the limits on the isolation ratio provided by the current seismic codes for base isolation (e.g., MIT 2018; CEN 2004), are motivated by the need to separate the dynamic behaviour of the two structural portions; lower $r \omega$ values would result in the interaction between the deformations of the superstructure and the substructure, reducing the effectiveness of the isolation system, and therefore the overall structural performance. Instead, the

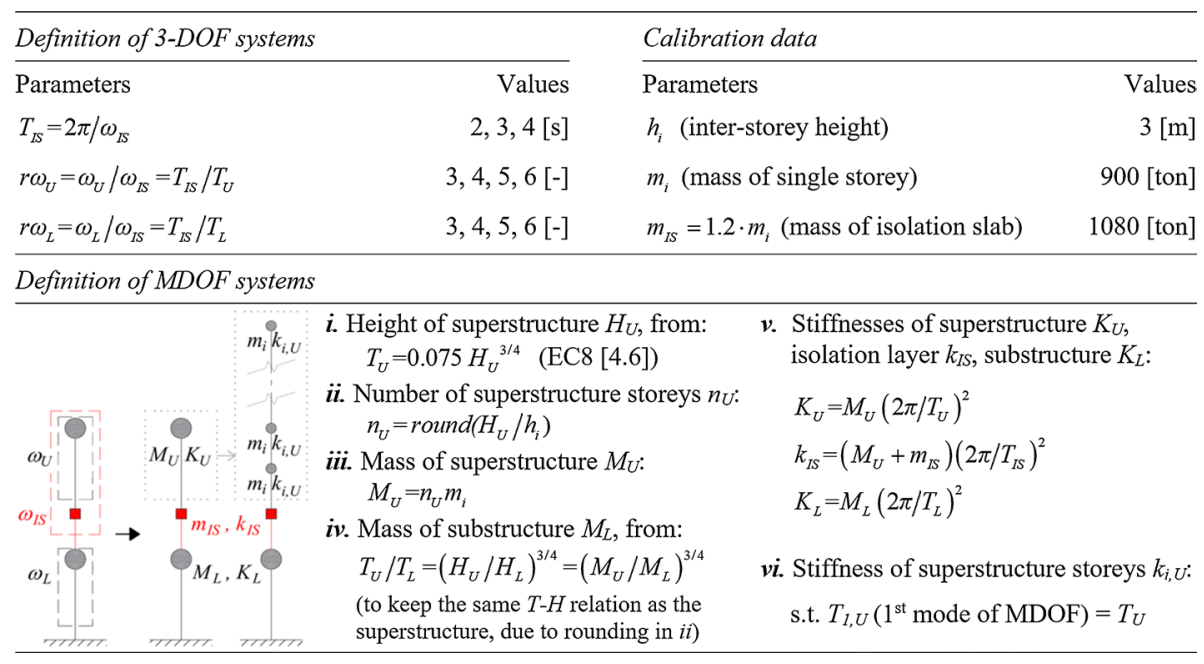

Fig. 1 Parametric analysis data and procedure for defining MDOF models 
maximum values of $r \omega_{U}$ and $r \omega_{L}$ (equal to six) have been used to define structures with a significant number of storeys, i.e., greater than or equal to two. In fact, as shown in Table 2, the number of storeys of the superstructure (and ideally of substructure) ranges from two to 15 . The range of parameters chosen then allows to avoid the phenomenon of modal coupling, which is responsible for dynamic amplifications of the structural response; this phenomenon occurs when the frequency ratio between one mode of the substructure and a higher mode of the isolated superstructure approaches one (see Faiella and Mele 2019). In addition, the mass ratio between the total isolated mass and the mass of the substructure varies from 0.5 to three, covering a fairly wide range of cases for this type of application.

Structural damping was defined as described above, using the Rayleigh model (Eq. 3) for the substructure and the stiffness-proportional model (Eq. 4) for the superstructure. As previously discussed, the latter model tends to overestimate the damping ratios $\zeta_{i}$ for higher modes, and therefore must be carefully calibrated. To this end, and to define the damping parametrically and objectively for various case studies, the superstructure was provisionally modelled as an equivalent 2-DOF system (derived similarly to the MDOF system), thus obtaining a global 4-DOF model where the first mode refers to isolation deflection, the second one to substructure deformation and third and fourth to superstructure deformation. Therefore, the damping coefficients for the substructure were calibrated as in Eq. 21, associating $\zeta=5 \%$ with the frequencies of modes two and four of the 4-DOF model, which define a sufficiently wide range of frequencies for the substructure of these case studies (even though its modal contribution is given almost completely by the second mode). Whereas, the damping coefficient for the superstructure was calibrated as in Eq. 22, associating $\zeta=5 \%$ with the frequency of mode four of the 4-DOF model, which is always between the third and fourth mode frequencies of the associated MDOF system.

$$
\begin{gathered}
\alpha_{L}=\zeta \cdot \frac{2 \omega_{i} \omega_{j}}{\omega_{i}+\omega_{j}}=0.05 \cdot \frac{2 \omega_{2} \omega_{4}}{\omega_{2}+\omega_{4}} ; \beta_{L}=\zeta \cdot \frac{2}{\omega_{i}+\omega_{j}}=0.05 \cdot \frac{2}{\omega_{2}+\omega_{4}} \\
\beta_{U}=\zeta \cdot \frac{2}{\omega_{k}}=0.05 \cdot \frac{2}{\omega_{4}}
\end{gathered}
$$

Figure 2 shows the $\zeta_{i}$ values, obtained with this approach and calculated by Eq. 5 , for the first four modal frequencies of all the analysed MDOF models. As expected, these values are around $5 \%$ for the deformation modes of the substructure $\left(\omega_{2}\right)$ and superstructure $\left(\omega_{3-4}\right.$, from three to six $\left.\%\right)$, whereas they are almost zero for the first mode $\left(\omega_{1}\right.$, the structural deformation being negligible in this mode).

Table 2 Number of storeys of superstructure (and ideally of substructure) for the various case studies

\begin{tabular}{lccll}
\hline$T_{I S}$ & $r \omega=3$ & $r \omega=4$ & $r \omega=5$ & $r \omega=6$ \\
\hline 2 & 6 & 4 & 3 & 2 \\
3 & 11 & 7 & 5 & 4 \\
4 & 15 & 11 & 8 & 6 \\
\hline
\end{tabular}



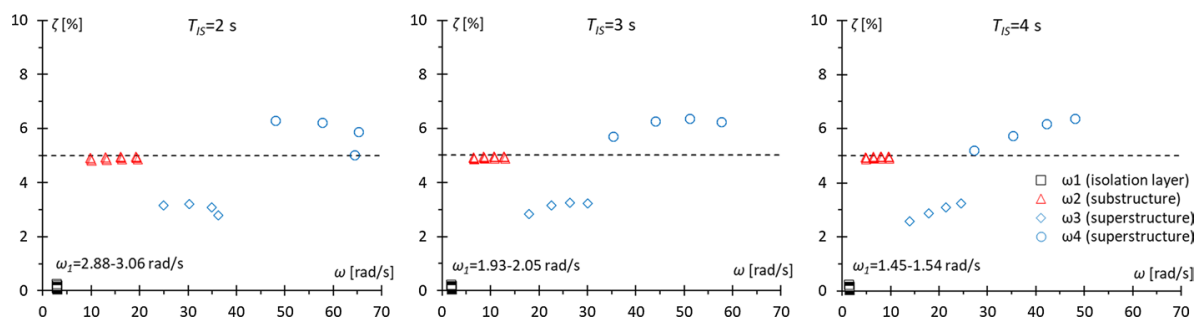

Fig. 2 Structural damping ratios $\left(\zeta_{i}\right)$ for the first four modal frequencies $\left(\omega_{i}\right)$ of all the MDOF models

\subsection{Seismic isolation systems}

All previously defined structural models were analysed with two types of isolation systems: linear system with fuse behaviour (fuse system) and non-linear system with LRBs (LRB system).

As already discussed, the restoring force $\mathrm{F}_{\mathrm{IS}}(t)$ of the isolation system is given in general by two contributions: one linear $\mathrm{F}_{\mathrm{IS}-l}(t)$, due to a linear or post-yielding stiffness $\left(k_{\mathrm{IS}}\right)$, and one hysteretic $\mathrm{F}_{\mathrm{IS}-h}(t)$, associated with both elastic $(k)$ and post-yielding $\left(k_{\mathrm{IS}}\right)$ stiffnesses.

A completely linear system provides the linear contribution $\mathrm{F}_{\text {IS- } l}(t)$ only, as shown in Eq. 23; this system is the simplest one, but it is inadequate to withstand non-seismic lateral service loads, and therefore not feasible.

$$
\mathrm{F}_{\mathrm{IS}-l}(t)=k_{\mathrm{IS}} x(t)
$$

Instead, the fuse system overcomes this problem by adopting mechanical fuse restraints, i.e., devices with stiffness and resistance values such as to exclude the operation of the isolation system for lateral service loads, and allow it for seismic loads, breaking some sacrificial components upon reaching a certain force or displacement $\left(d_{y}\right)$ threshold. This system also provides a linear restoring force, but changes its stiffness due to the breakage of the disposable elements, as Eq. 24 shows:

$$
\left\{\begin{array}{l}
\mathrm{F}_{\mathrm{IS}-l}(t)=k x(t) \text { until } d<d_{y} \\
\mathrm{~F}_{\mathrm{IS}-l}(t)=k_{\mathrm{IS}} x(t) \text { thereafter }
\end{array}\right.
$$

The LRB system provides both linear and hysteretic contributions. This system was chosen among the non-linear ones currently available because it is widespread in China, where the interest in inter-storey isolation is high. In this case, the restoring force is modelled using the Bouc-Wen model (Ismail et al. 2009), according to Eqs. 7 and 8, repeated in Eqs. 25 and 26 for convenience.

$$
\begin{gathered}
\mathrm{F}_{\mathrm{IS}}(t)=\mathrm{F}_{\mathrm{IS}-l}(t)+\mathrm{F}_{\mathrm{IS}-h}(t)=k_{\mathrm{IS}} x(t)+\left(k-k_{\mathrm{IS}}\right) d_{y} z(t)=k_{\mathrm{IS}} x(t)+Q z(t) \\
\dot{z}(t)=\left(A \dot{x}(t)-\beta|\dot{x}(t)| \cdot z(t) \cdot|z(t)|^{n-1}-\gamma \dot{x}(t)|z(t)|^{n}\right) / d_{y}
\end{gathered}
$$

The $Q$ parameter in Eq. 25 represents the characteristic damping force, corresponding to the intersection of the generic force-displacement loop with the force axis (at $x=0$ ). In general, isolation systems are designed to be activated for forces ranging from five to $15 \%$ of the isolated weight; lower values could cause movements for service loads or excessive 
displacements. In this study, considering also the resistance and dissipation provided by the FVDs, $Q$ was set as the lower limit, as shown in Eq. 27 (where $g$ is the gravity constant), as this allows the best seismic performance. The linear or post-yielding stiffness $k_{\text {IS }}$ depends on the isolation period $T_{I S}$ according to Eq. 28, whereas the elastic stiffness $k$ depends on $Q, k_{\mathrm{IS}}$ and $d_{y}$, as shown in Eq. 29. The yielding displacement $d_{y}$ was set as $10 \mathrm{~mm}$-a reference value identified in the catalogues of the main suppliers of LRB devices. This value was also used to define the change in stiffness of the fuse system (Eq. 24) to consistently compare the isolation technologies. Finally, the dimensionless parameters in Eq. 26 were assumed as in Liu et al. (2018), i.e., $A=\beta=\gamma=1$ and $n=2$; these values allow a good fit with some experimental force-displacement loops, provided in Kalpakidis and Constantinou (2008, p.132), representative of a fairly wide range of applications.

$$
\begin{gathered}
Q=5 \% \cdot\left(M_{U}+m_{I S}\right) \cdot g \\
k_{\mathrm{IS}}=\left(M_{U}+m_{I S}\right) \cdot\left(\frac{2 \pi}{T_{I S}}\right)^{2} \\
k=\frac{Q}{d_{y}}+k_{I S} .
\end{gathered}
$$

\subsection{Seismic inputs}

The dynamic equation of motion in Eq. 14 was solved for eight natural accelerograms, chosen from among those in the European Strong-Motion Database (ESD, Ambraseys et al. 2002). They were scaled to be compatible, on average, with the following elastic response spectrum of EC8: Type $1, a_{g}=0.25 \mathrm{~g}$ (bedrock acceleration), soil B (i.e., soil factor $S=1.2$, acceleration plateau between $T_{B}=0.15 \mathrm{~s}$ and $T_{C}=0.5 \mathrm{~s}$, and $T_{D}=2.0 \mathrm{~s}$ ). The peak ground acceleration PGA $\left(=a_{g} \cdot S\right)$ is $0.3 \mathrm{~g}$. The main details of the assumed natural records and their scale factors are reported in Table 3 . The associated acceleration and displacement

\begin{tabular}{|c|c|c|c|c|c|c|c|}
\hline Ref. & Earthquake & Location & Date & $\mathrm{M}_{\mathrm{w}}$ & $\begin{array}{l}\text { Fault mecha- } \\
\text { nism }\end{array}$ & $\begin{array}{l}\text { Distance from } \\
\text { epicentre }(\mathrm{km})\end{array}$ & Scale factor \\
\hline Acc. 1 & $\begin{array}{l}\text { Montenegro } \\
\text { (aftershock) }\end{array}$ & Montenegro & $1979 / 05 / 24$ & 6.2 & thrust & 20 & 5.42 \\
\hline Acc. 2 & $\begin{array}{r}\text { Campano } \\
\text { Lucano }\end{array}$ & Italy & $1980 / 11 / 23$ & 6.9 & normal (y) & 33 & 3.02 \\
\hline Acc. 3 & Erzincan & Turkey & 1992/03/13 & 6.6 & strike slip & 13 & 0.59 \\
\hline Acc. 4 & Ano Liosia & Greece & 1999/09/07 & 6.0 & normal & 18 & 3.51 \\
\hline Acc. 5 & $\begin{array}{r}\text { Campano } \\
\text { Lucano }\end{array}$ & Italy & $1980 / 11 / 23$ & 6.9 & normal (x) & 33 & 3.04 \\
\hline Acc. 6 & Tabas & Iran & $1978 / 09 / 16$ & 7.3 & oblique & 57 & 1.71 \\
\hline Acc.7 & Ano Liosia & Greece & 1999/09/07 & 6.0 & normal & 20 & 0.32 \\
\hline Acc. 8 & Montenegro & Montenegro & $1979 / 04 / 15$ & 6.9 & thrust & 25 & 0.66 \\
\hline
\end{tabular}
spectra are shown in Fig. 3, compared with the EC8 spectrum.

Table 3 Information on the assumed accelerograms (from ESD database, Ambraseys et al. 2002) 

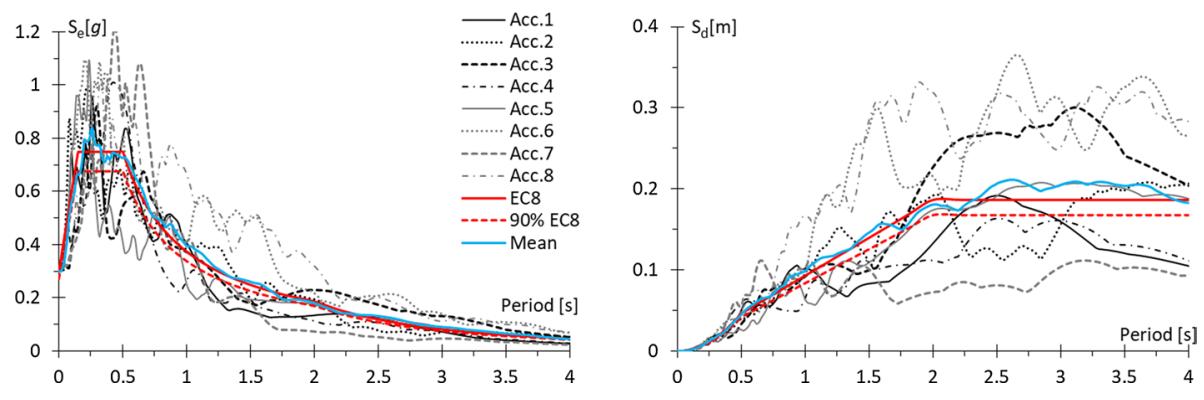

Fig. 3 Acceleration and displacement response spectra compatible with the EC8 spectrum

\section{Results of the parametric analysis}

\subsection{Structural behaviour with additional FVDs}

For preliminary assessments, Fig. 4 shows the TH responses of some performance parameters for the case study: $T_{I S}=3 \mathrm{~s}, r \omega_{U}=r \omega_{L}=3$, and Acc.1; this figure compares the cases without FVD and with linear $(\alpha=1)$ and non-linear $(\alpha=0.2)$ FVDs, with $\zeta_{F V D, l}=0.3$ (i.e., $\left.r_{F} \approx 0.5\right)$, separately for the two isolation systems. The performance parameters are drift of isolation layer, drift of superstructure (i.e., displacement of top floor relative to isolation layer), and absolute acceleration of top storey.

The following considerations can be drawn:

- As expected, with the same additional damping ratio $\zeta_{F V D, l}\left(\right.$ or force ratio $r_{F}$ ), the maximum drift of the isolation layer is greater for the fuse system, especially without FVD.

- The use of FVD with the fuse system significantly changes the vibration frequencies, which decrease for the isolation layer and increase for the superstructure. Instead, no significant variations in frequency are noted for the LRB system, which is less sensitive to the use of FVD.

- FVD is clearly effective in reducing the isolation drift for both isolation systems, whereas its effects on the superstructure are not easily predictable.

- The linearity degree of the FVD seems to significantly influence the structural response. In case studies of Fig. 4, non-linear FVDs $(\alpha=0.2)$ are more effective than linear ones $(\alpha=1)$ in reducing the isolation drift, but tend to amplify more the superstructure response, particularly for the fuse system.

For the same case studies as in Figs. 4 and 5 shows the force-displacement loops of isolators and dampers separately. Thus, the following considerations can be drawn:

- With the same additional damping ratio $\zeta_{F V D, l}$ (or force ratio $r_{F}$ ), the response with the LRB system is characterised by smaller isolator deflections (as seen in Fig. 4) and smaller damper forces, due to the presence of hysteretic damping in addition to the viscous one.

- Non-linear FVDs behave more rigidly than linear ones, with high dissipative forces even for small velocities (as noted in the range $\pm 20 \mathrm{~mm}$ ), as they are associated with greater damping coefficients $c_{n l}$ for the same force ratio $r_{F}$. This different behaviour 
influences the structural response, more for the fuse system than the LRB system (as seen in Fig. 4).

In general, $\alpha$ seems a significant parameter for structural response optimisation, and what has been seen justifies the need for an optimisation study.
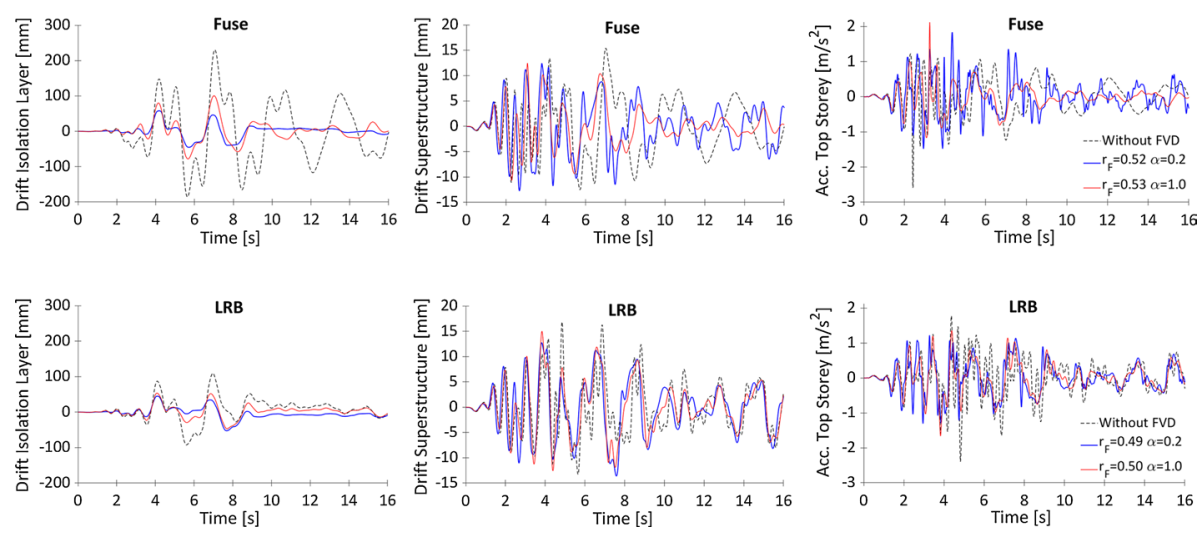

Fig. $4 \mathrm{TH}$ responses of structural performance parameters of a case study structure $\left(T_{I S}=3 \mathrm{~s}\right.$, $\left.r \omega_{U}=r \omega_{L}=3\right)$, for both isolation systems and Acc.1: cases without FVD and with linear $(\alpha=1)$ and nonlinear $(\alpha=0.2)$ FVDs $\left(r_{F} \approx 0.5\right)$
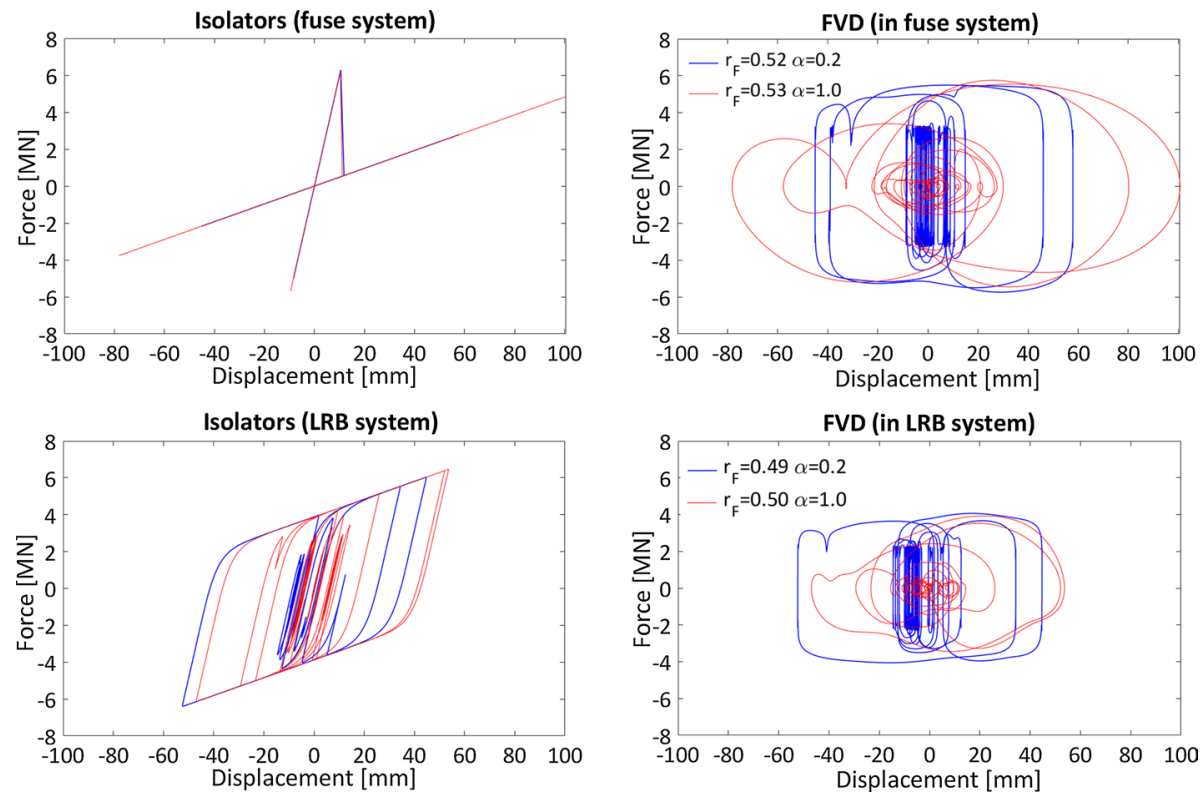

Fig. 5 Force-displacement loops of isolators (left) and dampers (right) for the same case studies (with FVD) as in Fig. 4 


\subsection{Peak response surfaces with additional FVDs}

To globally assess the additional damping effects on the structural response for all the examined FVDs, the peak values of the various performance parameters recorded in the $\mathrm{TH}$ analyses (averaged between the accelerograms) were plotted and linearly interpolated against the FVD variables, $\alpha$ and $r_{F}$, obtaining the corresponding peak response surfaces. Figures 6, 7 and 8 show and compare these response surfaces for some case studies, with reference to the following performance parameters: drift of isolation layer $D_{I S}$, maximum inter-storey drift of superstructure $D_{U}$, maximum absolute floor acceleration of superstructure $A_{U}$.

In particular, Fig. 6 compares the response surfaces of the structure $T_{I S}=3 \mathrm{~s}$ and $r \omega_{U}=r \omega_{L}=6$ between the isolation systems. The main observations are reported below:

- $D_{I S}$ is strongly influenced by the maximum damper force $\left(r_{F}\right)$, but only slightly by $\alpha$. In particular, $D_{I S}$ is reduced as $r_{F}$ increases, and this reduction is more evident
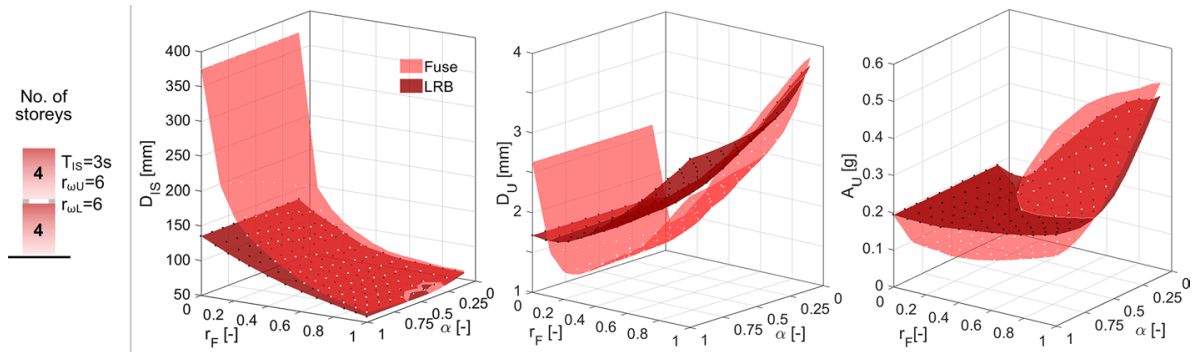

Fig. 6 Response surfaces of the structure $T_{I S}=3 \mathrm{~s}, r \omega_{U}=r \omega_{L}=6$ : comparison between isolation systems
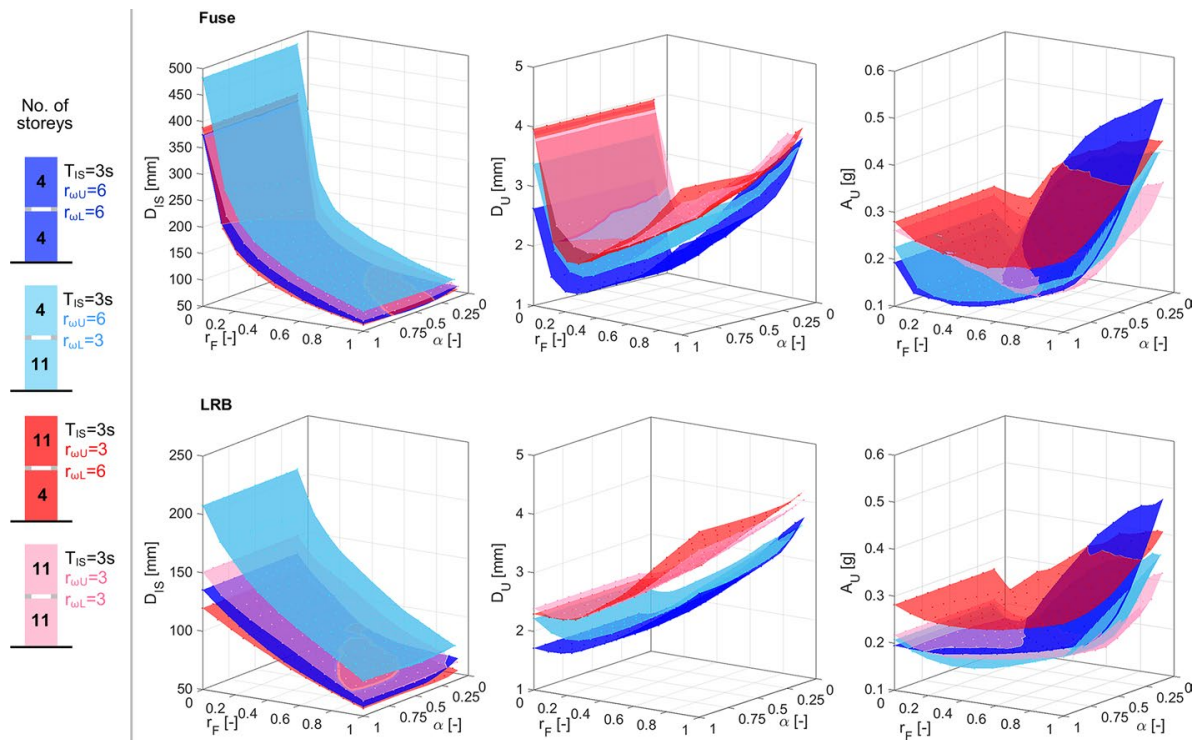

Fig. 7 Response surfaces for $T_{I S}=3 \mathrm{~s}$, fuse (above) and LRB (below) isolation systems: various $r \omega_{U}$ and $r \omega_{L}$ 

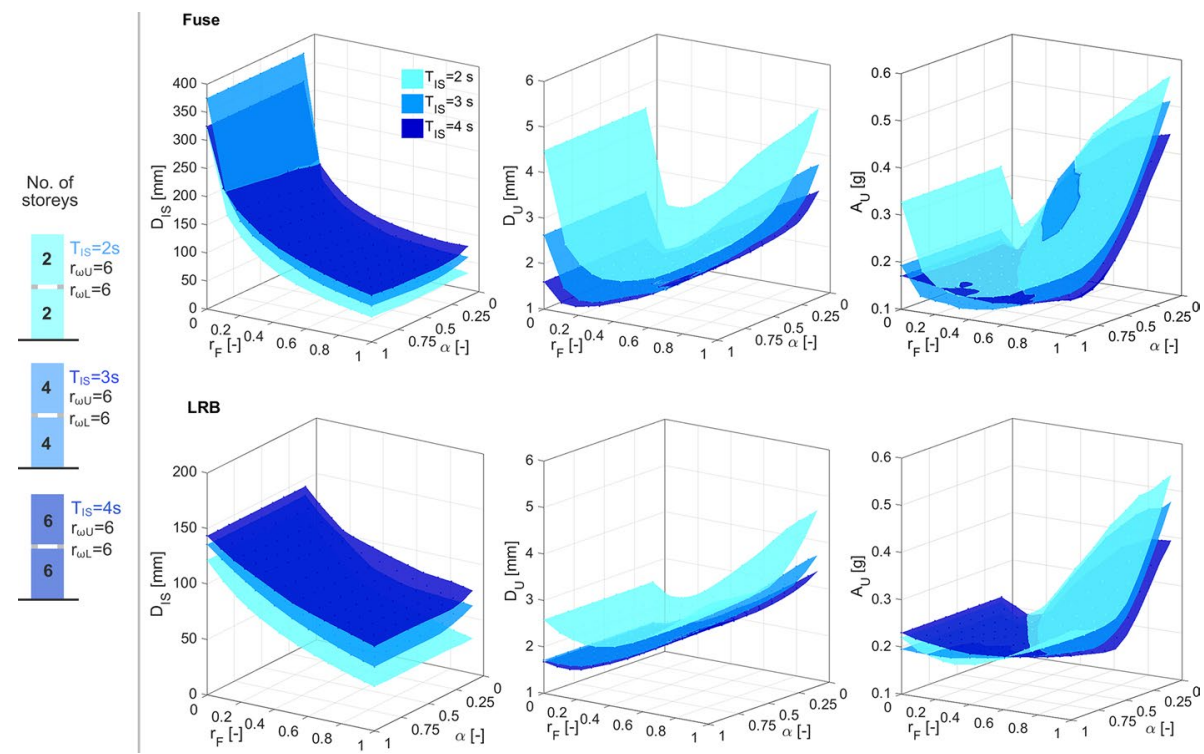

Fig. 8 Response surfaces for $r \omega_{U}=r \omega_{L}=6$, fuse (above) and LRB (below) isolation systems: various $T_{I S}$

for the fuse system, which shows a drift at $r_{F}=0$ much greater than that of the LRB system (as seen in Fig. 4).

- $D_{U}$ is generally influenced by both FVD parameters. In particular, the dependence on $\alpha$ increases with increasing $r_{F}$ (for highly dissipative solutions) and the greater the non-linearity (rigidity) of the FVD, the greater the amplification of $D_{U}$. For small $r_{F}$ values (up to 0.25 for the case studies shown), the increase in damper force considerably reduces $D_{U}$ for the fuse system and only slightly influences $D_{U}$ for the LRB system, due respectively to the absence and presence of further dissipation provided by the isolators. Similarly to $D_{I S}$, the fuse system exhibits a much greater drift at $r_{F}=0$ than that of the LRB system.

- The influence of $r_{F}$ and $\alpha$ on the parameter $A_{U}$ is somewhat similar to that observed for $D_{U}$. However, $A_{U}$ seems to be less influenced (flatter trend) than $D_{U}$ for low $r_{F}$ values and linear or nearly linear FVDs (more effective in containing accelerations). Also, for such dampers, these accelerations are smaller for the fuse system (as expected).

- In general, the structural responses with the two isolation systems tend to coincide for high $r_{F}$ values and low $\alpha$ values (increasing both the force and rigidity of the FVD).

- Although $D_{I S}$ can be similarly minimised for the two isolation systems (with high damping values), the superstructure response $\left(D_{U}, A_{U}\right)$ can be reduced more in the case of the fuse system, providing only a viscous damping. In this situation, however, the associated $D_{I S}$ values turn out to be greater than those of the LRB system; hence, it is needed to evaluate and compare multiple optimal solutions.

Figure 7 compares the response surfaces of the structures with $T_{I S}=3 \mathrm{~s}$ and $r \omega_{U^{-}}$ $r \omega_{L}=6-6,3-6,6-3,3-3$ (corresponding to structures of 4-4, 11-4, 4-11 and 11-11 storeys respectively, see Table 2), separately for the fuse and LRB systems. Similar colours represent cases with the same superstructure (blue for the more rigid one $r \omega_{U}=6$, red for 
the more flexible one $r \omega_{U}=3$ ) and similar intensities represent cases with the same substructure (dark for $r \omega_{L}=6$, light for $r \omega_{L}=3$ ). The main observations are reported below:

- For all these structures, the trend of the surfaces is like that of Fig. 6 for the same isolation system.

- For both the systems, $D_{I S}$ increases as $r \omega_{L}$ decreases (from dark to light surfaces), i.e., when the substructure becomes more flexible. Then, with the same substructure, $D_{I S}$ increases as $r \omega_{U}$ increases (from red to blue surfaces), i.e., when the isolated mass becomes smaller, the latter being effective in containing the substructure displacements (acting as TMD). Therefore, $D_{I S}$ is reduced for low-rise substructures and significant isolated masses-solutions that resemble the base isolation.

- For both isolation systems, $D_{U}$ and $A_{U}$ increase as $r \omega_{U}$ decreases (from blue to red surfaces), i.e., when the superstructure becomes more flexible. With the same superstructure, the increase of $r \omega_{L}$ (darker surfaces) reduces $D_{U}$ for low damping values (especially for the fuse system), but could increase it for high damping solutions, as the limit situation of base isolation (for large $r \omega_{L}$ and small $r_{F}$ ) becomes that of fixed-base superstructure (for large $r \omega_{L}$ and large $r_{F}$ ); then, the increase of $r \omega_{L}$ generally amplifies $A_{U}$, especially for high $r_{F}$ and low $\alpha$ values (rigid FVDs).

Figure 8 compares the response surfaces of the structures with $r \omega_{U}=r \omega_{L}=6$ and $T_{I S}$ $=2,3$ and $4 \mathrm{~s}$ (corresponding to structures of 2-2, 4-4 and 6-6 storeys respectively, see Table 2), separately for the fuse and LRB systems. For all $T_{I S}$ values, the trend of the surfaces is like that of Fig. 6 for the same isolation system. In general, as $T_{I S}$ increases, $D_{I S}$ increases whereas $D_{U}$ and $A_{U}$ decreases (as expected); furthermore, with increasing $T_{I S}$, the variability of $D_{U}$ and $A_{U}$ on the analysed $r_{F}-\alpha$ range is reduced, especially for the fuse system.

Figures 6, 7 and 8 clearly show that the damping solutions that minimise the peak response of the various performance parameters are identified in different $r_{F}-\alpha$ ranges; hence, a multi-objective design procedure is needed to determine a set of Pareto-optimal solutions. Moreover, the trend of $D_{U}$ is quite similar to that of $A_{U}$ for optimisation purposes and is generally more sensitive to the additional damping for low to medium $r_{F}$ values, where the superstructure peak response is at its minimum. Therefore, to solve the optimisation problem in a simpler way, without significantly affecting the results, the superstructure performance can be effectively represented only by $D_{U}$ for these case studies. Globally, linear or quasi-linear FVDs seem more effective in reducing the isolation drift while containing the amplification of the superstructure response.

\section{Optimisation of the structural performance and optimal FVDs}

\subsection{Surrogate response models and genetic algorithm}

To apply the proposed optimisation method, appropriate surrogate response models should be defined and calibrated, based on previously obtained response surfaces. These models can be effectively expressed in a totally dimensionless form by normalising the structural response to the peak one in the case without damper, thus allowing the comparison of the optimisation results between various case studies. The performance parameters chosen for optimisation are the relative displacement of the isolation layer 
$\left(D_{I S}\right)$ and maximum inter-storey drift of the superstructure $\left(D_{U}\right)$. Defining $r D_{I S}$ and $r D_{U}$ as the associated parameters normalised to the case without FVD, the two objective functions (OFs) to be minimised simultaneously are as follows:

$$
\left\{\begin{array}{l}
O F_{1}=\min \left[r D_{I S}\left(r_{F}, \alpha\right)\right] \\
O F_{2}=\min \left[r D_{U}\left(r_{F}, \alpha\right)\right]
\end{array}\right.
$$

where, $r D_{I S}\left(r_{F}, \alpha\right)$ and $r D_{U}\left(r_{F}, \alpha\right)$ are the surrogate response models of $r D_{I S}$ and $r D_{U}$, obtained by calibrating the complete fourth degree polynomial in $r_{F}$ and $\alpha$ on the response surfaces of interest, separately for each case study examined. The polynomial degree was chosen based on preliminary assessments of computational time and prediction capability, the latter described by the coefficient of determination $R^{2}$. The values of $R^{2}$, averaged among the case studies analysed, are shown in Fig. 9 as a function of the polynomial degree, separately for the performance parameters $\left(r D_{I S}\right.$ and $\left.r D_{U}\right)$ and the two types of isolation systems. Ongoing studies by the same authors suggest the appropriateness of this polynomial model, even in the case of irregular structures (i.e., with different mass and stiffness distribution between substructure and superstructure).

The NSGA-II algorithm (Deb et al. 2002) was used to solve the multi-objective optimisation problem and its parameters were set as specified in Table 4, based on computational cost-effectiveness assessments. The high number of generations and large population (number of solutions sought) allow for a refined solution and were possible due to the surrogate models. Indeed, the minimisation of analytical functions requires much shorter calculation times than the direct optimisation of the structural response, based on the TH analysis iteration (as done by Liu et al. 2018).

Given the large population, a filter was then applied to the optimal results for representation purposes. This filter returns the mean values of the OFs for regular $r_{F}$ intervals and associates them with the central values of these intervals (the optimal $r_{F}$ range was divided into 25 intervals).

As an example, for the case study $T_{I S}=4 \mathrm{~s}, r_{\omega U}=r_{\omega L}=6$ and fuse system, Fig. 10a shows the $r D_{I S}$ and $r D_{U}$ surrogate response models and Fig. 10b the application of the

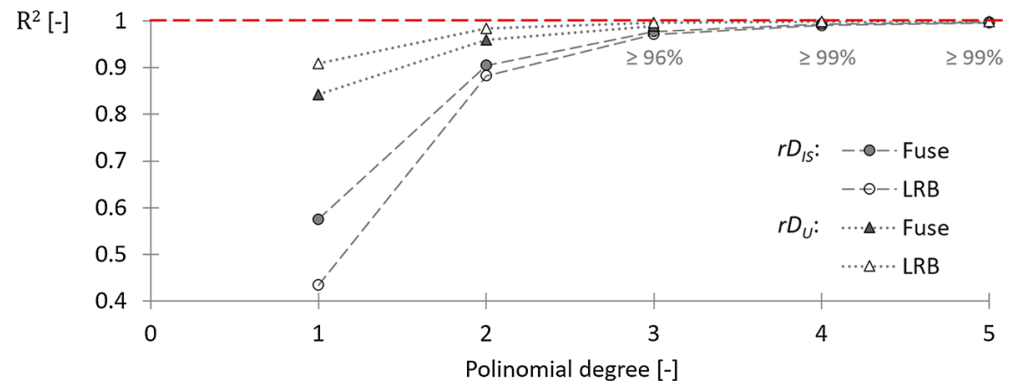

Fig. 9 Accuracy of polynomial surrogate response models as a function of their degree, in terms of $\mathrm{R}^{2}$ averaged among the case studies

Table 4 Parameters of the NSGA-II algorithm

\begin{tabular}{llll}
\hline Number of generations & Population size & Crossover probability & Mutation probability \\
\hline 200 & 500 & 0.9 & 0.1 \\
\hline
\end{tabular}




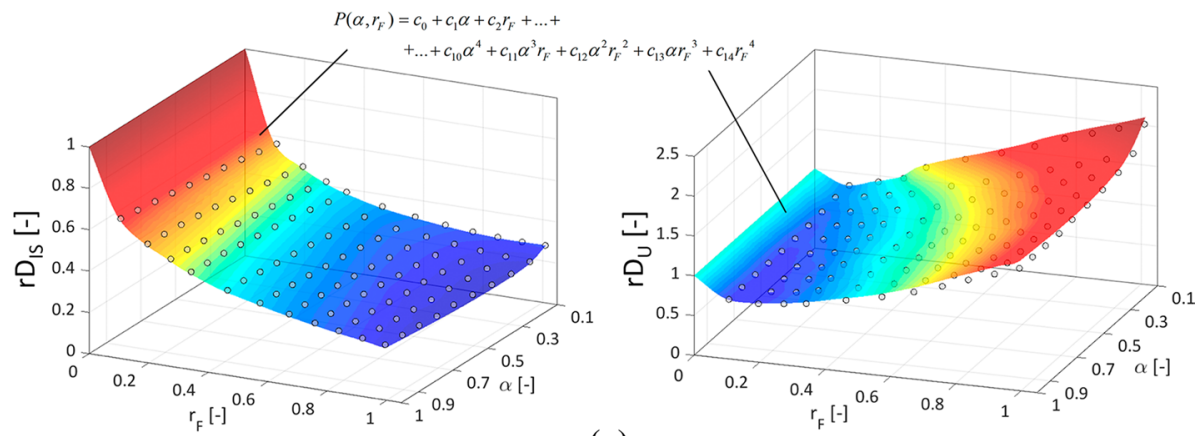

(a)
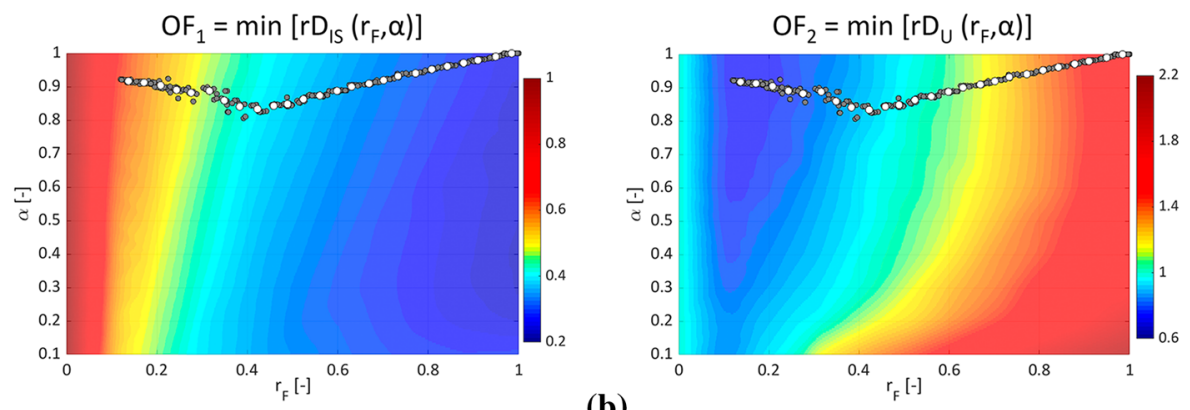

(b)

Fig. 10 a Surrogate response models of $r D_{I S}$ and $r D_{U}$ calibrated for the case: $T_{I S}=4 \mathrm{~s}, r_{\omega U}=r_{\omega L}=6$, fuse system. b Colour maps of the surrogate models and optimisation results $\left(O F_{1}\right.$ and $\left.O F_{2}\right)$ from NSGA-II

NSGA-II algorithm; in particular, in Fig. 10b, the optimal results of $O F_{1}$ (minimisation of $r D_{I S}$ ) and $O F_{2}$ (minimisation of $r D_{U}$ ) are superimposed on the surfaces of surrogate models (represented in $2 \mathrm{D}$ by a colour map), with grey markers representing the entire population and white for the filtered results. This representation clearly shows that $O F_{1}$ and $O F_{2}$ are minimised respectively, for the major and minor $r_{F}$ values, and the intermediate results are the best compromise solutions between OFs.

Furthermore, for design purposes, it is essential to determine the $c$ values associated with the optimal $r_{F}$ - $\alpha$ solutions; therefore, a 4-degree polynomial function was also calibrated to obtain the model of $c^{*}$ (Eq. 20), as a function of $r_{F}$ and $\alpha$, from which the optimal values of $c$ can be derived.

\subsection{Optimisation results}

The overall optimisation results are shown in Figs. 11 and 12 for the fuse and LRB systems respectively, as optimal values of OFs, $\alpha$ and $c^{*}$ versus $r_{F}$.

Regarding the OFs, the following considerations can be drawn:

- $O F_{1}$ is only slightly influenced by the type of structure. In particular, with the same $r_{F}, O F_{1}$ slightly increases with increasing $T_{I S}$, as the $D_{I S}$ values generally increase with $T_{I S}$ (see Fig. 8), and so do their ratios with respect to the case without damper (i.e., $O F_{1}$ ). For the same $T_{I S}$, the negligible dependence of $O F_{1}$ on the type of struc- 

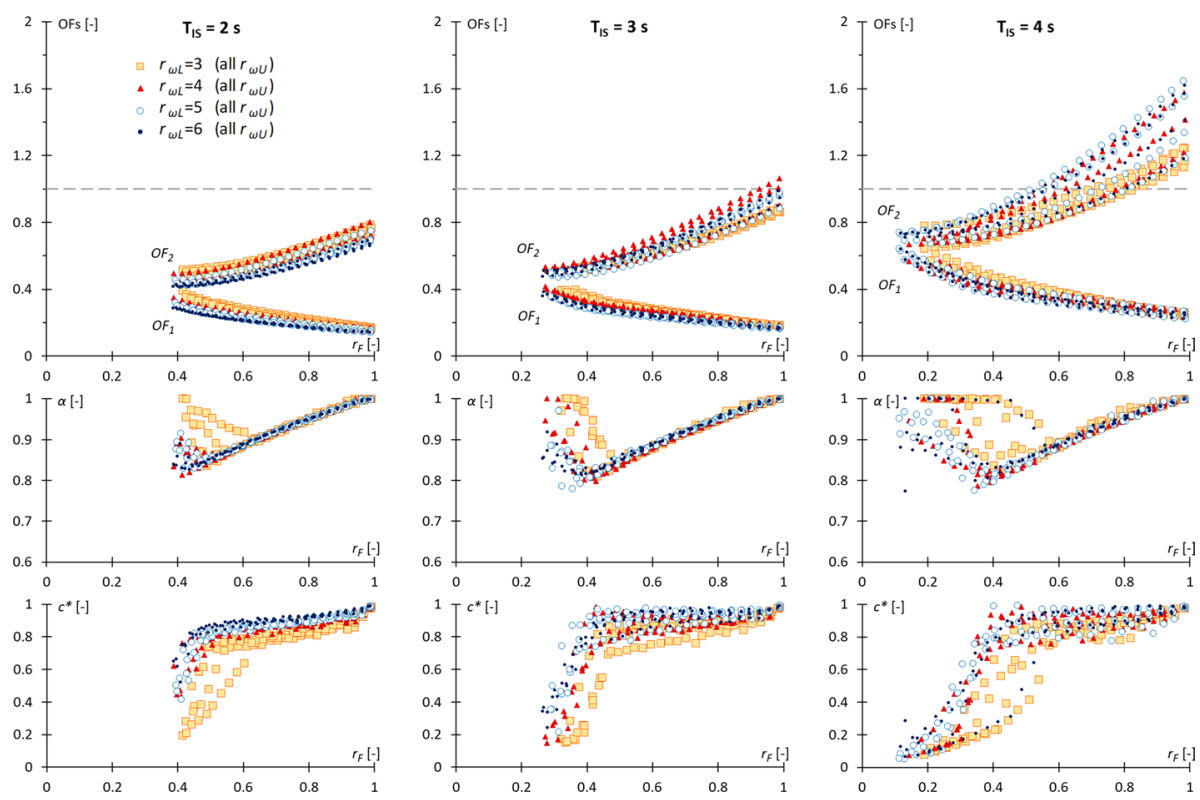

Fig. 11 Optimal results of OFs, $\alpha$ and $c^{*}$, versus $r_{F}$, for all the case studies with the fuse isolation system
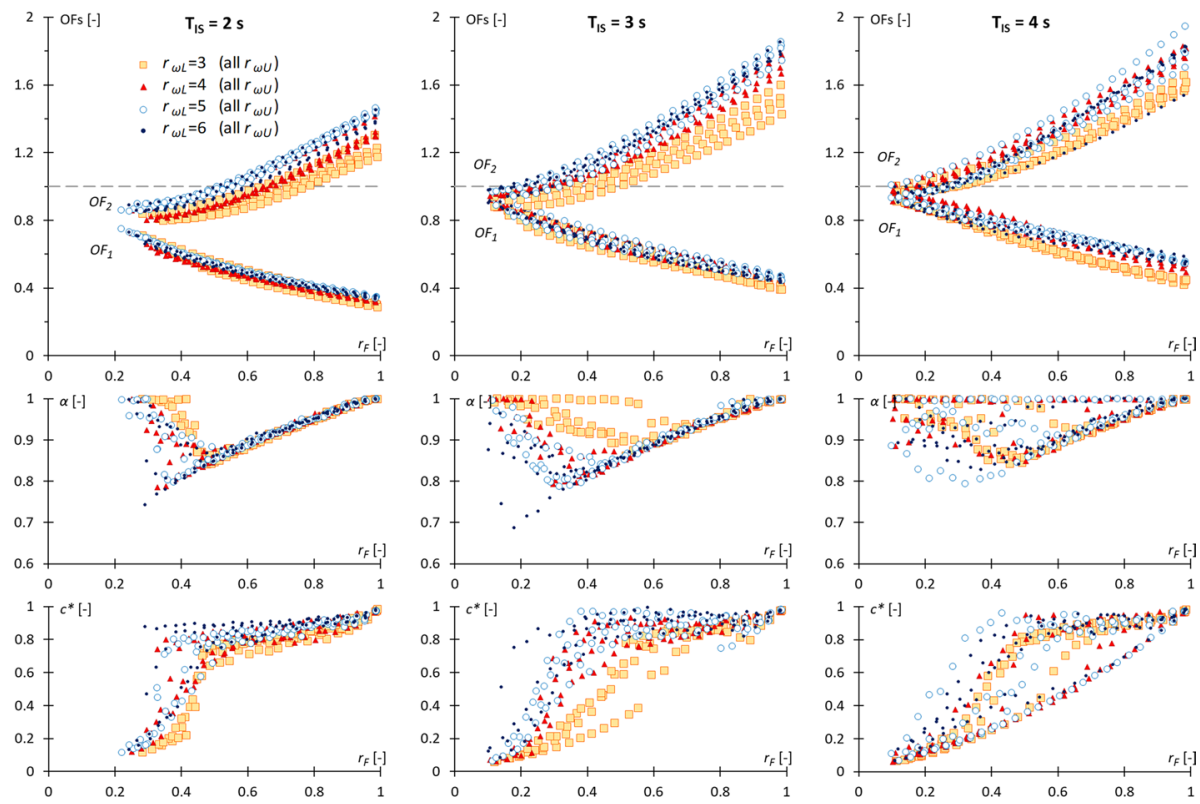

Fig. 12 Optimal results of OFs, $\alpha$ and $c^{*}$, versus $r_{F}$, for all the case studies with the LRB isolation system

ture indicates the effectiveness of $r_{F}$ in representing a characteristic force ratio for the structure (being defined based on the first vibration mode). Indeed, a given $r_{F}$ value corresponds to different FVD forces for different structures, but such as to 
provide a similar reduction of the first-mode seismic response (and so of $D_{I S}$ and $\left.O F_{1}\right)$.

- Regarding $O F_{2}$, its dependence on the type of structure is more evident. In particular, when $T_{I S}$ increases, the minimum values of $r_{F}\left(r_{F m i n}\right)$ associated with the minimum values of $O F_{2}$ are reduced, and the values of $O F_{2}$ increase globally as well as their dependence on both $r_{F}$ and the type of structure. This is explained by the fact that $D_{U}$ (hence $O F_{2}$ ) is a parameter influenced by the amplification effects of higher modes, which clearly depend on the type of structure and are more important, in relative terms, for higher $T_{I S}$ values (see Fig. 8).

Regarding the FVD parameters, the following considerations can be drawn:

- The optimal $\alpha$ values are distributed along $r_{F}$ with a quasi-triangular trend-first they decrease to a minimum value $\left(\alpha_{\text {min }}\right)$ for incremental $r_{F}$ values from low to medium, then they rise up to one for higher $r_{F}$ values. This trend seems generally correlated with that of $O F_{2}$, where for $r_{F}<r_{F}\left(\alpha_{\text {min }}\right), O F_{2}$ increases only slightly from its minimum value, whereas its increase is significant for larger $r_{F}$. Considering that in general, $O F_{1}$ is already significantly reduced at $r_{F}\left(\alpha_{\min }\right)$ and the damper cost increases with increasing $r_{F}$, the optimal solution associated with $\alpha_{\min }$ represents a significant design reference and convenient lower limit for $r_{F}$. However, for the slenderest structures (minor $r_{\omega U}$ and $r_{\omega L}$ and major $T_{I S}$ ), and particularly for the LRB system, this trend of $\alpha$ is less evident and tends to flatten out on the value of $\alpha=1$ (linear FVDs).

- For the significant $r_{F}$ values i.e., $r_{F} \geq r_{F}\left(\alpha_{m i n}\right), \alpha$ is independent of the type of structure. This is another advantage of using $r_{F}$ (similar to what observed for $O F_{1}$ ). Instead, the values of $\alpha_{\text {min }}$ (from about 0.75 to 0.9 ) and $r_{F}\left(\alpha_{\text {min }}\right)$ (from about 0.3 to 0.6) generally depend on the type of structure as well as the results for $r_{F}<$ $r_{F}\left(\alpha_{\text {min }}\right)$, because they concern the minimisation of $O F_{2}$ (which is influenced by the type of structure). However, $\alpha$ variability is limited for the regular structures analysed.

- To increase the damper force $\left(r_{F}\right)$ between $r_{F m i n}$ and $r_{F}\left(\alpha_{m i n}\right), c^{*}$ increases strongly, as the optimal $\alpha$ values are reduced in this range; once $r_{F}\left(\alpha_{\min }\right)$ is reached (with $c^{*}$ values from about 0.65 to 0.95 ), the increase of $r_{F}$ continues with only a slight increase of $c^{*}$, and up to one for $r_{F}=1$ (for all structures), as it is supported by the increase in $\alpha$. Furthermore, it can be observed that variations in the trend of $c^{*}$ clearly reflect those in the trend of $\alpha$.

The trend of the optimal solutions is similar between the isolation systems, but the values of $O F_{1}$ and $O F_{2}$ are greater for the LRB system. This is due to the fact that the reference values of $D_{I S}$ and $D_{U}$ in the case without damper (the normalisation values for $O F_{1}$ and $O F_{2}$ ) are lower for this system, because of the hysteretic damping. Therefore, the results in this dimensionless form cannot be used directly to make considerations about the convenience of the two systems, which will be discussed in the next section.

As a reference, assuming $r_{F} \approx 0.4$ (fairly representative of the solutions with $\alpha_{\text {min }}$ ) and for $T_{I S}$ from 2 to $4 \mathrm{~s}, O F_{1}$ ranges from 0.3 to 0.5 for the fuse system and from 0.5 to 0.9 for the LRB system, and $O F_{2}$ ranges from 0.4 to 0.9 for the fuse system and from 0.9 to 1.2 for the LRB system. 


\subsection{Global assessment of structural performance with optimal FVDs}

This section presents the absolute values of the main performance parameters $\left(D_{I S}, D_{U}\right.$ and $A_{U}$ ) for all the optimal solutions obtained, in order to draw some considerations about the effectiveness of the additional damping, also in relation to the type of isolation system.

The maximum inter-storey drift ratio of the substructure $\left(\delta_{L}\right)$ was also evaluated. In particular, the assumed structural models represent the substructure as an equivalent single degree of freedom (ESDOF), and therefore do not allow directly evaluating $\delta_{L}$. However, based on the ESDOF displacement $\left(D_{L, E S D O F}\right)$, and the hypothesis of a linear deflection profile along the substructure height $\left(H_{L}\right.$, calculated as in Fig. 1$)$, it was possible to estimate $\delta_{L}$ through Eq. 31 (which, not considering the modal participation factor, slightly overestimates $\delta_{L}$ ).

$$
\delta_{L} \approx \frac{3}{2} \frac{D_{L, E S D O F}}{H_{L}}
$$

The values of these parameters are plotted in Fig. 13 versus $r_{F}$ for all optimal solutions. For convenience, $D_{U}$ was also shown as a ratio with respect to the storey height $\left(h_{i}\right)$, i.e., $\delta_{U}=D_{U} / h_{i}$. The following considerations can be drawn:

- $D_{I S}$ is significantly reduced by increasing $r_{F}$ up to values between four and $12 \mathrm{~cm}$ for $r_{F}=1$ (for all case studies), with an almost asymptotic trend for higher $r_{F}$ values (this reduction is consistent with the results in Liu et al. 2018). For the fuse system, the values of $D_{I S}$ are initially higher but decrease more rapidly, tending to the values of the LRB system for high $r_{F}$ values.

- Although $\delta_{U}$ increases with increasing $r_{F}$, its values remain reasonably low for the stock of regular structures analysed, i.e., less than $0.2 \%$ for the entire range of $r_{F}$. These values are lower for the fuse system and unlike $D_{I S}$, this trend is maintained throughout the $r_{F}$ range.

- The values of $A_{U}$, not directly optimised in the OFs, show a similar trend to that of $\delta_{U}$, with values generally lower for the fuse system. Then, the optimal damping solutions allow containing these accelerations below $0.3 \mathrm{~g}$ in most cases, which is generally an acceptable value to avoid significant damage to building contents.

- Regarding $\delta_{L}$, also not optimised in the OFs, the optimal FVDs allow a good control of the substructure response, limiting these drifts to $0.5 \%$ in all cases-a value generally assumed in the structural codes (e.g., EC8) as damage limit state. Furthermore, considering that these drifts are slightly overestimated as well, this result confirms the adequacy of linear modelling for these substructures.

Clearly, the structural modelling approach depends on the type of structures examined, and the hypothesis of elastic substructure may not be appropriate for some applications. For example, when inter-storey isolation is used as a seismic retrofit technique for existing buildings, the evaluation of the possible and specific non-linear behaviour of the substructure is generally necessary.

These results also allow some considerations on the convenience of isolation systems. For $r_{F}$ values from low to medium in the analysed range (less than about 0.7 ), the two systems can lead to similar structural performances, i.e., similar values of $D_{I S}$ and $\delta_{U}$, even if with higher $r_{F}$ values for the fuse system. Therefore, the choice of the isolation type could 

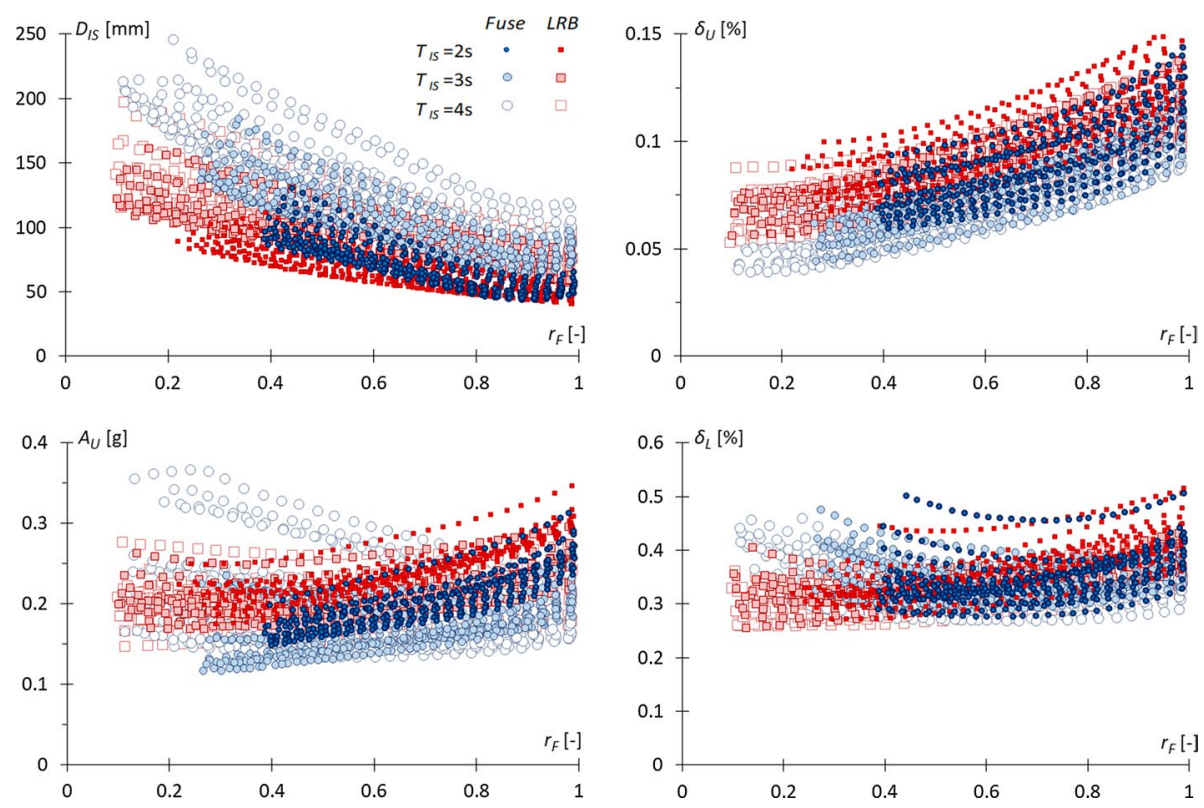

Fig. 13 Main performance parameters $\left(D_{I S}, \delta_{U}, A_{U}\right.$ and $\left.\delta_{L}\right)$, versus $r_{F}$, for all optimal solutions

be based on specific cost assessments (beyond the scope of this paper), considering the difference in cost between isolators (to the advantage of the fuse system) and dampers (to the advantage of the LRB system, requiring a minor $r_{F}$ for the same performance). Instead, for higher $r_{F}$ values and particularly for low $T_{I S}$ values, i.e., when the will is to strongly limit the inter-storey deflection, the fuse system allows lower $\delta_{U}$ and $A_{U}$ values for the same $D_{I S}$ values, and therefore a better structural performance at a lower cost (due to cheaper isolators with equal dampers).

Finally, these findings acquire further importance if considered as a reference or basis of comparison for future studies aimed at examining irregular structures (where the effects of additional damping can be much greater), substructures with non-linear behaviour (which require specific non-linear modelling) or different seismic inputs (e.g., near-fault earthquakes).

\subsection{Optimisation of linear FVDs and comparisons}

The optimisation results obtained with $\alpha_{\min } \approx 0.8$ indicate that the optimal FVDs for the analysed regular structures are slightly non-linear. Therefore, it is interesting to understand the extent to which this non-linearity influences the structural performance $\left(O F_{1}, O F_{2}\right)$. To this end, the proposed optimisation method was reapplied to all case studies, setting $\alpha=1$.

The results obtained are interesting, as they show that this non-linearity only slightly influences the structural response with respect to the case of optimal linear FVDs. In particular, as shown in Fig. 14 for both the fuse and LRB systems, the ratios between the values of $O F_{1}$ previously obtained and those obtained with linear optimisation $\left(O F_{1, L}\right)$ are all greater than 0.92 , i.e., the structural performance in terms of $O F_{1}$ is reduced to a maximum 

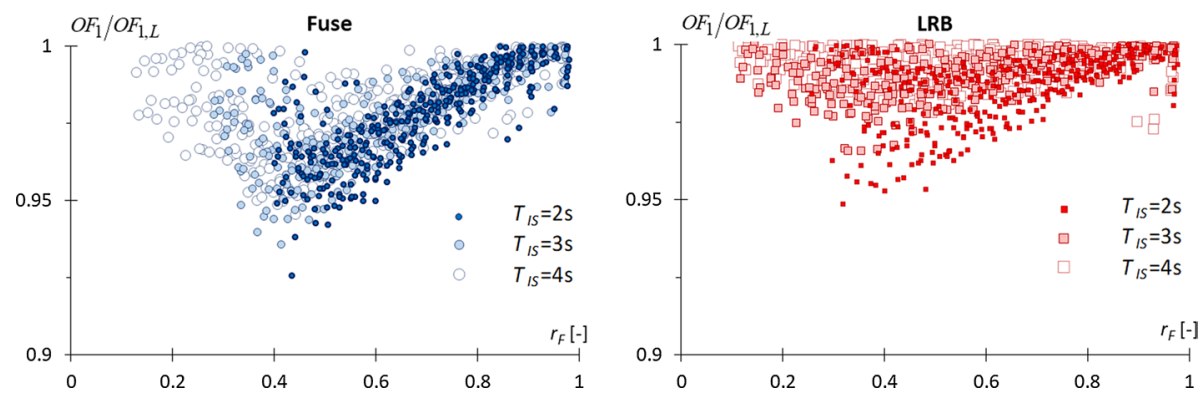

Fig. 14 Ratio between the $O F_{1}$ values and the associated ones of $O F_{1, L}$ from the optimisation of linear FVDs
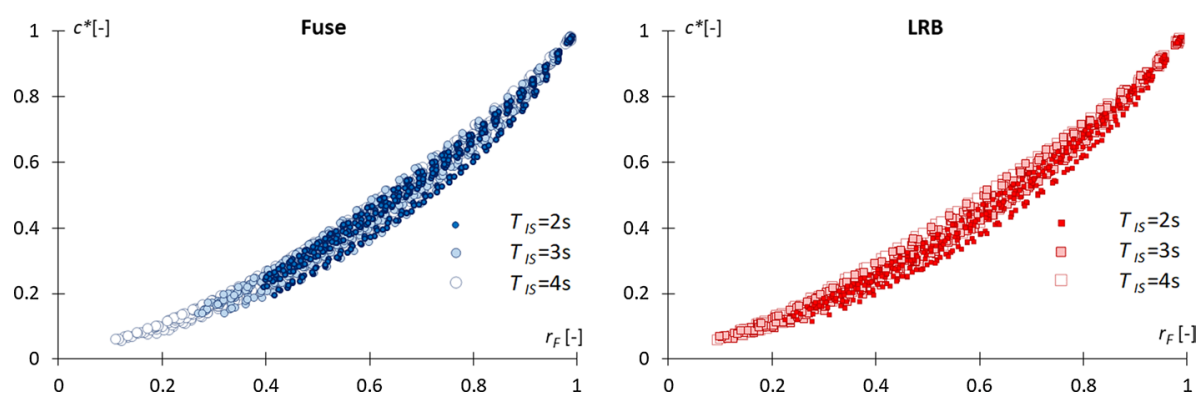

Fig. 15 Optimal values of $c^{*}$, versus $r_{F}$, from the optimisation of linear FVDs

of $8 \%$ (in the case of fuse system). Moreover, the same variation for $\mathrm{OF}_{2}$ is negligible, as contained in the range $\pm 4 \%$.

Figure 15 shows the $c^{*}$ values for optimal linear FVDs and all case studies, separately for the fuse and LRB systems. With $\alpha=1, c^{*}$ (see Eq. 20) corresponds precisely to the first-mode damping ratio due to the damper, and its values show approximately the same trend for all structures with respect to $r_{F}$, again highlighting the effectiveness of $r_{F}$ for this study. Also, these $c^{*}$ values are very similar between the two isolation systems. In particular, the increase of $c^{*}$ with respect to $r_{F}$ is more than linear, because with increasing $r_{F}$, the damper velocity is reduced, and therefore to increase the damper force with constant $\alpha$ $(=1), c$ must increase more than proportionally.

\section{Prediction models for the optimal design of FVDs}

\subsection{Definition of prediction models}

Based on linear optimisation results, the best solution for design purposes would seem to be the use of linear FVDs. To this end, Fig. 16a, b show (in red) the prediction models assumed for the parameters of linear FVDs; in particular, $\alpha$ is always 1 (Fig. 16a) and $c^{*}$ is provided as a function of $r_{F}$ only (Fig. 16b), valid for both isolation systems. 


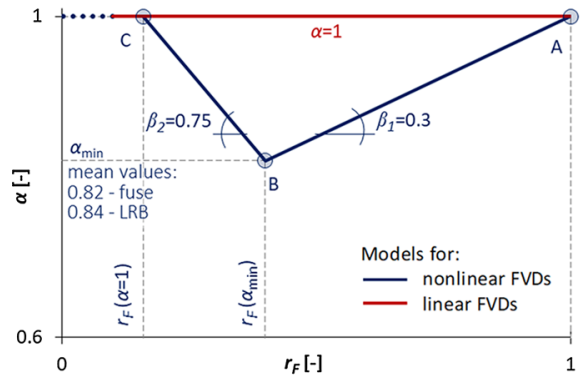

(a)

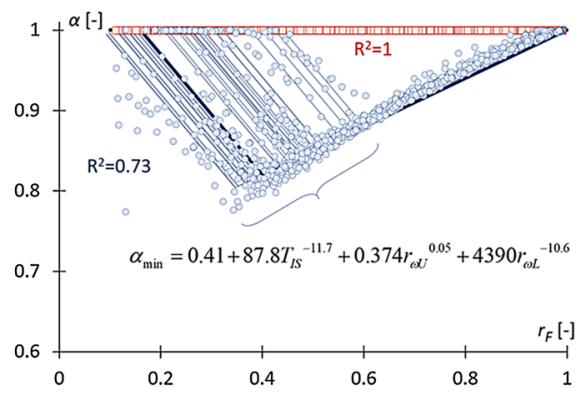

(c)

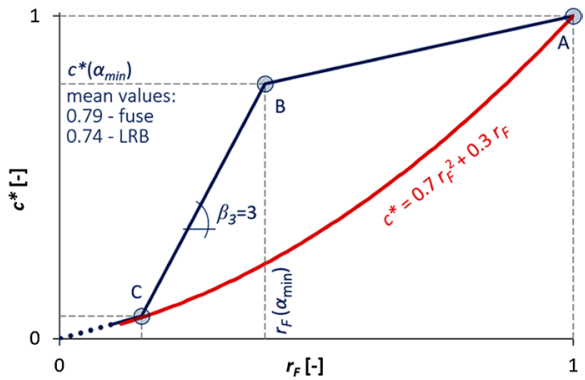

(b)

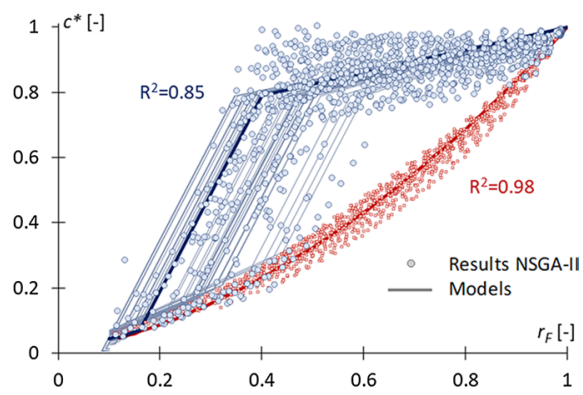

(d)

Fig. $16 \mathbf{a}$, b Prediction models of $\alpha$ and $c^{*}$, for optimal linear (red) and non-linear (blue) FVDs. c, d Fit of the models and associated values of $R^{2}$ for the case of the fuse system

However, it is also useful to provide the prediction models of $\alpha$ and $c^{*}$ on the basis of the general optimisation results, and therefore of the trends and dependencies observed in Figs. 11 and 12-these models are also represented in Fig. 16a, b (in blue).

In addition, for the fuse system, Fig. 16c, d show the fit of $\alpha$ and $c^{*}$ models, for both linear and non-linear dampers, and associated values of the coefficient of determination $\left(R^{2}\right)$. The models for non-linear FVDs were represented using both the average value of $\alpha_{\min }$ and for a more accurate estimate, the specific $\alpha_{\min }$ value of each structure predicted by the equation in Fig. 16c.

The non-linear models, besides predicting the real trend of the optimal FVD parameters and being a useful reference for future research work on different case studies, allow to identify the $r_{F}$ value associated with the $\alpha_{\min }$ point which, as previously discussed, represents an optimal compromise solution between $O F_{1}$ and $O F_{2}$. These models of $\alpha$ and $c^{*}$ are both tri-linear laws defined on three main points, whose $r_{F}$ values are in common for the two models. The points are: (A) $r_{F}=1, \alpha=c^{*}=1$; (B) position of $\alpha_{\text {min }}$; (C) intersection of model $\alpha$ with the line for $\alpha=1$. The calibration slopes $\left(\beta_{1}, \beta_{2}\right.$ and $\left.\beta_{3}\right)$ and mean values of $\alpha_{\min }$ and $c^{*}\left(\alpha_{\min }\right)$, necessary to determine the models, are shown in the relevant figures.

For clarity, the steps for applying these non-linear prediction models are listed below.

- Calculation of $\alpha$ by simple linear proportion, entering Fig. 16a with the chosen value of $r_{F}$ and using the slope $\beta_{1}$, if $r_{F} \geq r_{F}\left(\alpha_{\text {min }}\right)$ and $\beta_{2}$ vice versa. Based on the values of $\alpha_{\min }$ and $\beta_{1}$, shown in the same figure, the value of $r_{F}\left(\alpha_{\min }\right)$ that identifies the characteristic point B is 0.40 for the fuse system and 0.47 for the LRB system. 
- Calculation of $c^{*}$, similar to that of $\alpha$, entering Fig. 16b with the chosen value of $r_{F}$ and applying a linear proportion. Point B is defined by the same values of $r_{F}\left(\alpha_{\text {min }}\right)$ as reported above.

- Execution of the undamped modal analysis to determine the necessary information on the first vibration mode i.e., the first-mode critical damping $\left(c_{c r l}\right)$ and the first-mode isolation deflection $\left(\psi_{1, n L+1}-\psi_{1, n L}\right)$.

- Calculation of the damping constant $c$ of the FVD using Eq. 20, on the basis of $c^{*}$ and the first-mode characteristics previously obtained.

Regarding the choice of $r_{F}$, which depends on the structural performance sought, this can be based on the optimal results obtained for $O F_{1}$ and $O F_{2}$, shown in Figs. 11 and 12; in particular, as seen above, the globally most effective solutions are those with $r_{F} \approx r_{F}\left(\alpha_{\text {min }}\right)$.

In the following Sect. 6.2, for example and validation purposes, both linear and nonlinear prediction models were applied to three representative structures of the analysed stock, evaluating the structural performance with increasing $\mathbf{r}_{F}$ and the influence of damper non-linearity.

\subsection{Example of application of prediction models}

The models for predicting optimal FVDs were applied to the three case study structures represented in Fig. 17. These structures have 16 floors, with the isolation system placed

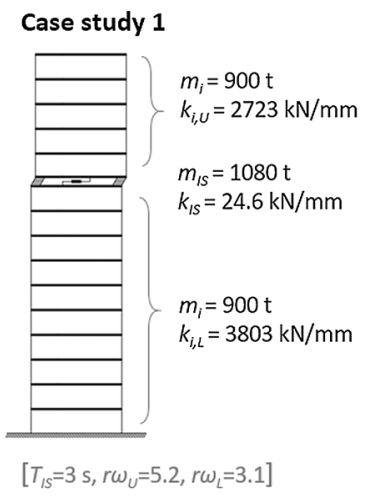

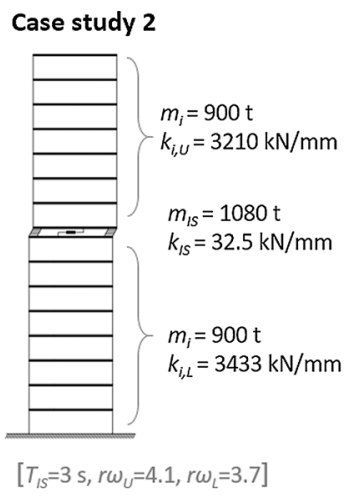

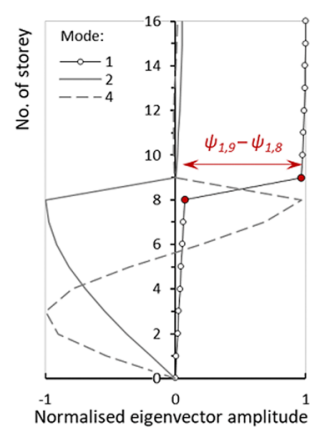

Case study 3

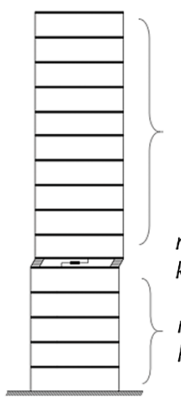

$m_{i}=900 \mathrm{t}$

$k_{i, U}=3803 \mathrm{kN} / \mathrm{mm}$

$m_{I S}=1080 \mathrm{t}$

$k_{l S}=44.5 \mathrm{kN} / \mathrm{mm}$

$m_{i}=900 \mathrm{t}$

$k_{i, L}=2723 \mathrm{kN} / \mathrm{mm}$

\begin{tabular}{|c|c|c|c|}
\hline \multicolumn{4}{|c|}{$\begin{array}{l}\text { Case study 2: } \\
\text { - modal analysis }\end{array}$} \\
\hline $\begin{array}{l}\text { No. } \\
\text { mode }\end{array}$ & $\begin{array}{c}\omega \\
{[\mathrm{rad} / \mathrm{s}]}\end{array}$ & $\begin{array}{l}\text { Modal } \\
\text { mass }[t]\end{array}$ & $\begin{array}{r}\mathrm{Mc} \\
\text { contrib }\end{array}$ \\
\hline 1 & 2.0 & 7215 & \\
\hline 2 & 11.8 & 3961 & \\
\hline 3 & 22.9 & 3856 & 0 \\
\hline 4 & 33.9 & 3877 & \\
\hline \multicolumn{4}{|c|}{ - first mode characteristics: } \\
\hline \multicolumn{4}{|c|}{$\psi_{1, n L+1}-\psi_{1, n L}=\psi_{1,9}-\psi_{1,8}=0.9$} \\
\hline \multicolumn{4}{|c|}{$c_{c r 2}=2 m_{1} \omega_{1}=28.9 \mathrm{KNs} / \mathrm{mm}$} \\
\hline
\end{tabular}

$\left[T_{1 S}=3 \mathrm{~s}, r \omega_{U}=3.1, r \omega_{L}=5.2\right]$

- equivalent 3-DOF system:

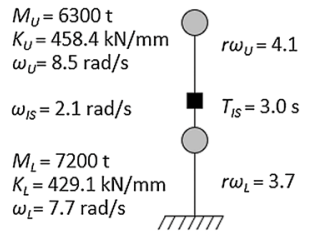

Fig. 17 Presentation of the case studies (above); modal analysis of the case study 2 (below) 
between the 10th and 11th floors for case study 1, between the eighth and ninth floors for case study 2, and between the fifth and sixth floors for case study 3. Both fuse and LRB isolation systems were analysed for each case study, with an isolation period $\mathrm{T}_{I S}=3 \mathrm{~s}$.

These structures were modelled as 16-DOF linear systems and their main structural data, as well as the characteristics of their equivalent 3-DOF systems (showing their representativeness with respect to the investigated parametric stock), are shown in Fig. 17. The same figure also shows the results of the classical modal analysis, performed on structure 2 , necessary for applying the prediction models.

In particular, for each structure and isolation system, the optimal non-linear FVD parameters were calculated for three levels of damper force, i.e., $r_{F}=0.2, r_{F}\left(\alpha_{\min }\right)$ and 0.8 , following the procedure described above. In the case of $r_{F}=r_{F}\left(\alpha_{\text {min }}\right)$, the parameter $c$ of the optimal linear FVD was also calculated for comparison purposes. Table 5 reports the optimal values of $c$ and $\alpha$ predicted for the various design solutions investigated.

To evaluate the effectiveness of the models, the structural responses without and with the identified FVDs were assessed by TH analysis, using the set of natural records and the structural damping previously defined. The main results are shown in Figs. 18 and 19. Figure 18 shows the inter-storey drift and absolute floor acceleration profiles of structure 2 for the selected $r_{F}$ values, obtained by averaging the maximum analysis values among the various accelerograms. Figure 19, on the other hand, summarises and compares the results of all case studies in terms of structural performance normalised to the case without FVD. In particular, the parameters evaluated are drift of isolation layer $\left(D_{I S}\right)$, maximum inter-storey drift of substructure $\left(D_{L}\right)$ and superstructure $\left(D_{U}\right)$, maximum absolute floor acceleration of substructure $\left(A_{L}\right)$ and superstructure $\left(A_{U}\right)$. These parameters, normalised to the case without FVD, provide $r D_{I S}, r D_{L}, r D_{U}, r A_{L}$ and $r A_{U}$, which are the parameters shown in Fig. 19.

As can be seen from both Figs. 18 and 19, the effects of the FVDs are clearly greater in the case of the fuse system, as it does not provide further dissipation. Then, for all the case studies, the FVD associated with $r_{F}\left(\alpha_{\text {min }}\right)$ proved to be the best compromise solution between the performances of the isolation layer and the substructure (both of which improve with increasing $r_{F}$, if not excessive) and that of the superstructure (which worsens with medium to high $r_{F}$ values).

In particular, the trends in Fig. 19 show that FVDs with $r_{F}>>r_{F}\left(\alpha_{\text {min }}\right)$, compared to the case of $r_{F}\left(\alpha_{\text {min }}\right)$, may allow a further reduction of $r D_{I S}$ and $r D_{L}$, however, at the expense of a more significant amplification of the superstructure response. Furthermore, very large $r_{F}$

Table 5 Optimal FVD parameters for case study structures, for three FVD force ratios $\left(r_{F}=0.2, r_{F}\left(\alpha_{\text {min }}\right)\right.$, $0.8)$

\begin{tabular}{|c|c|c|c|c|c|c|c|}
\hline \multirow[t]{2}{*}{ Isolation system } & \multirow{2}{*}{$\begin{array}{l}\text { FVD force ratio } \\
\mathrm{r}_{F}(-)\end{array}$} & \multicolumn{2}{|l|}{ Case study 1} & \multicolumn{2}{|l|}{ Case study 2} & \multicolumn{2}{|l|}{ Case study 3} \\
\hline & & $c\left[\mathrm{~N}(\mathrm{~s} / \mathrm{mm})^{\alpha}\right]$ & $\alpha(-)$ & $c\left[\mathrm{~N}(\mathrm{~s} / \mathrm{mm})^{\alpha}\right]$ & $\alpha(-)$ & $c\left[\mathrm{~N}(\mathrm{~s} / \mathrm{mm})^{\alpha}\right]$ & $\alpha(-)$ \\
\hline \multirow[t]{4}{*}{ Fuse } & 0.20 & 4938 & 0.97 & 6801 & 0.97 & 9535 & 0.97 \\
\hline & $0.40=r_{F}\left(\alpha_{\min }\right)$ & 20,531 & 0.82 & 28,278 & 0.82 & 39,647 & 0.82 \\
\hline & 0.40-linear & 6029 & 1 & 8305 & 1 & 11,643 & 1 \\
\hline & 0.80 & 24,169 & 0.94 & 33,290 & 0.94 & 46,672 & 0.94 \\
\hline \multirow[t]{4}{*}{ LRB } & 0.20 & 2052 & 1.00 & 2826 & 1.00 & 3962 & 1.00 \\
\hline & $0.47=r_{F}\left(\alpha_{\min }\right)$ & 19,274 & 0.84 & 26,547 & 0.84 & 37,219 & 0.84 \\
\hline & 0.47 -linear & 7683 & 1 & 10,582 & 1 & 14,836 & 1 \\
\hline & 0.80 & 23,455 & 0.94 & 32,305 & 0.94 & 45,292 & 0.94 \\
\hline
\end{tabular}



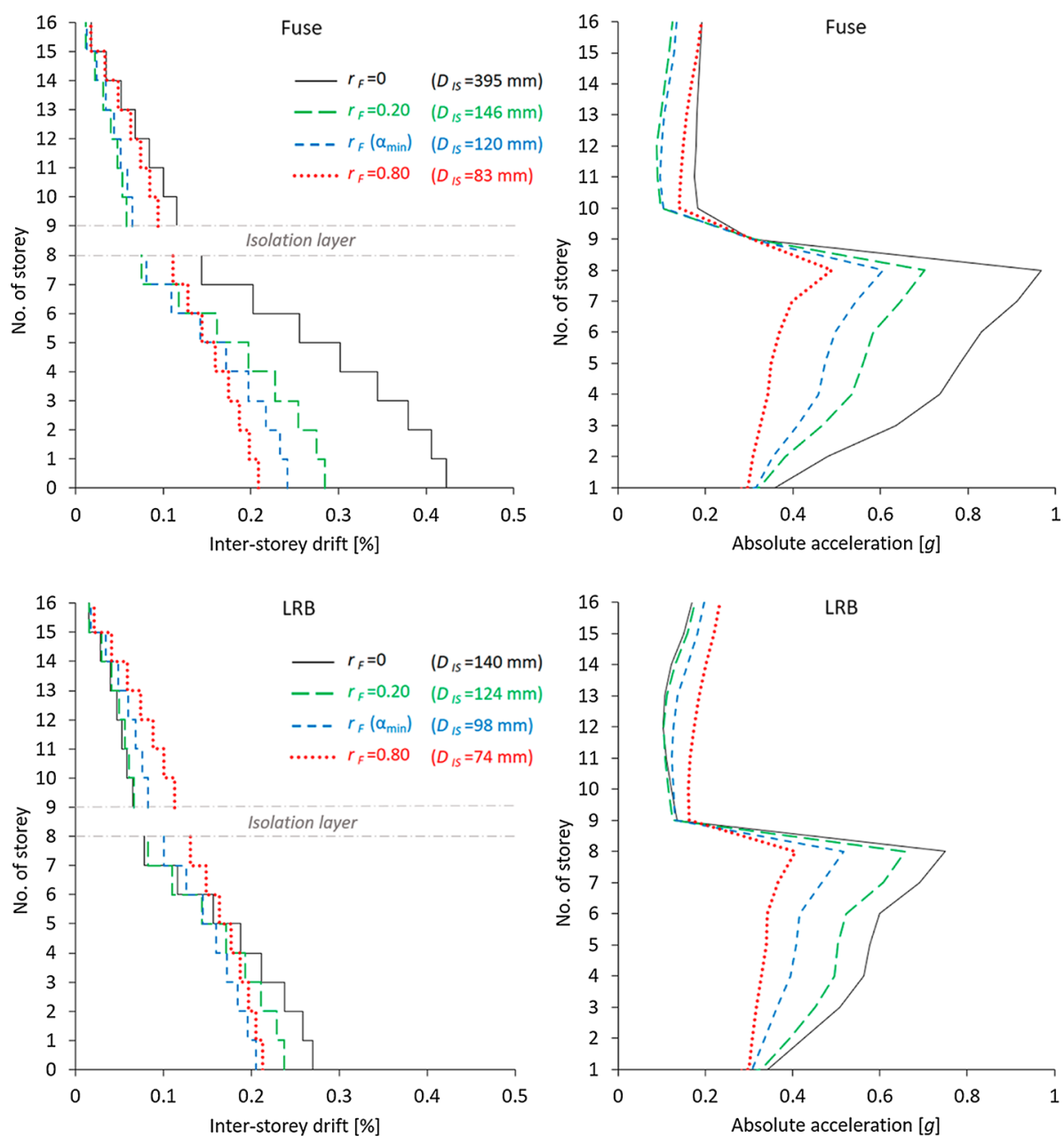

Fig. 18 Inter-storey drift and absolute floor acceleration profiles of the case study 2

values (such as 0.80) were also found to be less effective for the substructure in the case of the LRB system and a high isolated mass ratio (e.g., case study 3). Considering that these solutions are also the most expensive, they are generally to be avoided.

Figure 19 also shows the comparisons between optimal non-linear and linear FVDs for $r_{F}=r_{F}\left(\alpha_{\text {min }}\right)$. The related performance variations, in favour of non-linear dampers, are rather small for the fuse system and negligible for the LRB system, confirming the previous results of the parametric analysis.

It is also worth noting that as $r_{F}$ increases, both the inter-storey drift and floor acceleration profiles of the substructure are regularised along the building height, with the drift values decreasing in the lower part and increasing in the upper part of the substructure, and the acceleration values seeing an overall and considerable reduction (see Fig. 18).

Finally, with the aim of providing some preliminary indications on the possible effects of structural non-linearity, some TH analyses were carried out by modelling the structure of case study 2 with two non-linear models. These models are based on the hysteretic laws 


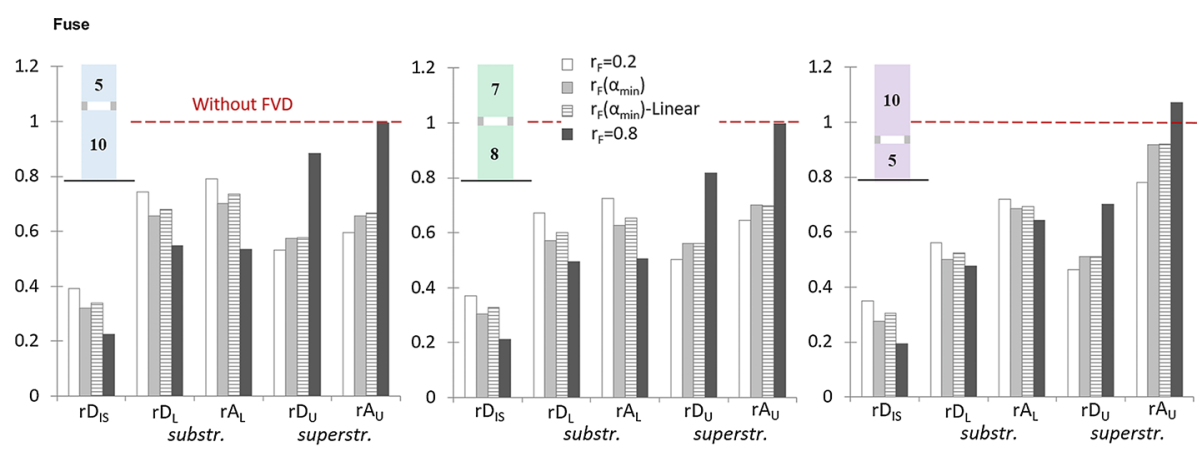

(a)
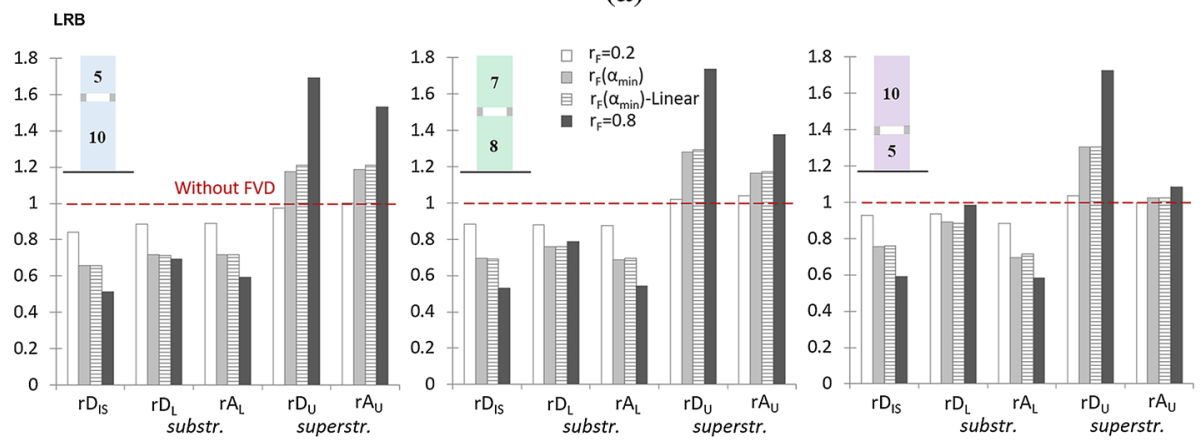

(b)

Fig. 19 Comparison of optimal structural performance with increasing $r_{F}$, and for $r_{F}=r_{F}\left(\alpha_{\min }\right)$ between optimal non-linear and linear FVDs, for case study structures with fuse (a) and LRB (b) isolation systems

of Takeda and Bouc-Wen, shown in Fig. 20a, which are often used to describe the behaviour of ductile RC frames. Both models were calibrated on the storey elastic stiffness $k_{i}$ (see Fig. 17), assuming a ratio $r$ between post-yield and elastic stiffnesses equal to 0.2, and an inter-storey drift $\delta_{y}$ at yield equal to $0.5 \%$. The Bouc-Wen model, which is based on a more complex hysteretic law (the same used to model LRB isolators), requires the calibration of additional hysteresis parameters, which were defined according to Sues et al. (1988) and are shown in Fig. 20a.

For the LRB isolation system, the FVD associated with $r_{F}\left(\alpha_{\min }\right)$, and the same set of accelerograms but evaluating two PGA values, Fig. 20b shows the inter-storey drift and absolute floor acceleration profiles, comparing the linear response of the structure with the non-linear ones associated with the two hysteretic models. For the PGA of $0.3 \mathrm{~g}$, the structure never exceeds $\delta_{y}$, thus no significant differences are observed. Instead, for the PGA of $1 \mathrm{~g}$, the substructure can yield, and therefore considerations on the effects of structural nonlinearity can be drawn. In particular, in the case of non-linear modelling, there is an amplification of the maximum drift values in the substructure, due to the concentration of plastic deformation on the lower storeys of the building, which first reach yield. This increase in deformation, and therefore in structural dissipation, then appears beneficial with regards to the floor accelerations, which can be significantly reduced. Clearly, the type of hysteretic model adopted plays a significant role. In the specific case study, the maximum values of drift and acceleration are lower using the Bouc-Wen model compared to the Takeda model, 


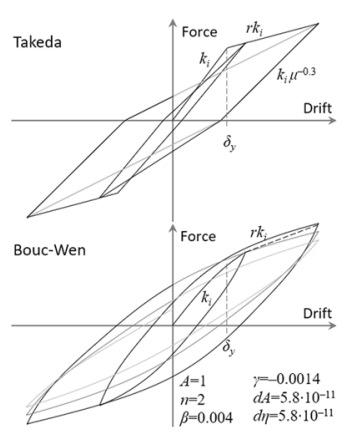

(a)
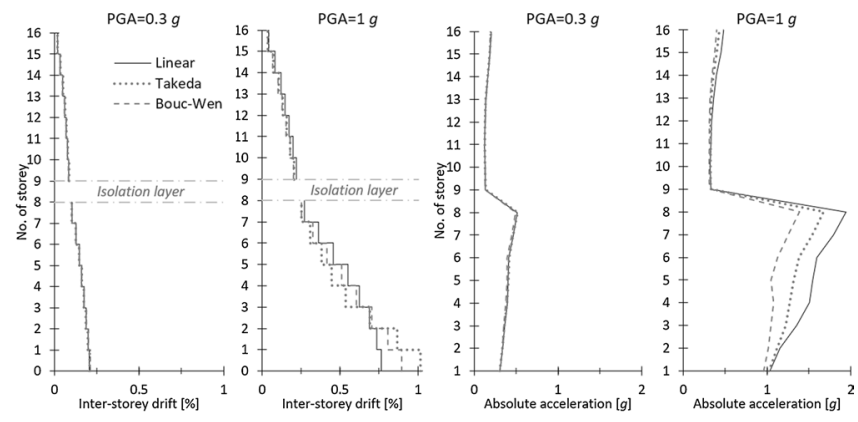

(b)

Fig. 20 a Non-linear structural models. b Performance comparisons between linear and non-linear modelling

as the former allows to describe the variation and degradation of the storey stiffness more gradually and also provides a slightly greater dissipation per cycle. The same considerations also apply to the fuse isolation system.

\section{Conclusions}

This paper aimed to evaluate the effects of optimal additional damping on the seismic performance of structures with inter-storey isolation. To this end, a new method for multiobjective optimisation and performance evaluation of FVDs was proposed. This method is based on the definition of appropriate surrogate response models, calibrated on the maximum response (averaged between seismic inputs) of some significant performance parameters and their subsequent minimisation through multi-objective genetic algorithms. Such models, if conveniently normalised, allow rationally comparing the FVD effects for a wide range of dampers and structures as well as obtaining optimisation results in a dimensionless form, suitable for the definition of prediction models.

This method was applied to a stock of 48 regular RC structures, with various vibration periods of superstructure, isolation and substructure, analysing a wide range of damping forces for both linear and non-linear dampers (with $\alpha$ between 0 and 1), two types of isolation systems (linear with fuse behaviour and non-linear with LRB isolators) and a set of spectrum-compatible natural records. The objective functions (OFs) evaluated in this optimal design, whose values were conveniently normalised to the case without damper, were the minimisation of the isolation drift $\left(O F_{1}\right)$ and the minimisation of the maximum inter-storey drift of the superstructure $\left(\mathrm{OF}_{2}\right)$. The maximum values of the superstructure floor accelerations and substructure inter-storey drifts were also evaluated as an output of the optimisation process. Furthermore, for comparison purposes, the proposed method was reapplied to optimise linear FVDs $(\alpha=1)$ only.

The main considerations on the comparative evaluation of the FVD effects are listed below:

- The values of $O F_{1}$ and $O F_{2}$ associated with optimal FVDs clearly showed a conflicting trend, proving the necessity of a multi-objective design to find a set of Pareto-optimal solutions. 
- For all the case studies, the optimal FVDs allowed significant reductions in isolation drift, with an even improved or only slightly amplified superstructure response (depending on the case study) for intermediate FVD force ratios $\left(r_{F}\right)$ among those analysed, confirming their effectiveness for inter-storey applications. Also, the values of $\mathrm{OF}_{2}$ were always less than two, with the greatest amplifications due to the higher values of $r_{F}$ and the isolation period.

- The optimal FVDs were slightly non-linear for the analysed structures, with minimum values of $\alpha$ ranging from about 0.75 to 0.9 .

- The optimal FVDs also ensured a good structural performance with respect to superstructure floor accelerations and substructure inter-storey drifts. For the case studies analysed, these accelerations were always less than $0.3 g$ and these drifts were always less than $0.5 \%$ (value generally assumed in the codes as damage limit state).

- The fuse isolation system was found to be slightly more advantageous than the nonlinear one with LRBs, when using very high $r_{F}$ values (greater than about 0.7 ), as it allowed better performance of the superstructure with the same reduction in isolation drift.

In addition, the main results from a design point of view are summarised below:

- A prediction model of optimal linear FVDs, calibrated on the results of the entire analysis stock, and a prediction model of optimal non-linear FVDs, calibrated separately for the fuse and LRB isolation systems, are provided in this paper.

- The optimal non-linear FVDs, compared to the linear ones with the same $r_{F}$ value, allowed a better structural performance, even if this benefit was rather limited for the analysed structures. Therefore, the use of the linear prediction model is the most practical solution from a design point of view. However, the non-linear models allowed to identify the $r_{F}$ value associated with the $\alpha_{\min }$ point, representing an optimal compromise solution between $O F_{1}$ and $O F_{2}$.

- For $r_{F} \approx r_{F}\left(\alpha_{\text {min }}\right)$ (i.e., 0.4 to 0.5), and compared to the case without FVD, the reductions in the isolation drift ranged from 50 to $75 \%$ for the fuse system and from 20 to $50 \%$ for the LRB system, considering all the case studies. On the other hand, the amplification of the maximum inter-storey drift of the superstructure (in the case of the LRB system) was always less than $20 \%$.

- The application of prediction models to specific case study structures confirmed their effectiveness and of optimal FVDs for these applications. Furthermore, some preliminary assessments on the effects of structural non-linearity showed how the latter influences the structural response by amplifying the inter-storey drifts in the lower part of the substructure and reducing floor accelerations.

The proposed method, due to its generality, can also be applied to different case studies from those examined here. For future work, it will be interesting to evaluate the effects and optimal parameters of the FVDs for irregular structures in elevation and compare them with those obtained in this study for regular structures, as well as examine different types of seismic input (e.g., near-fault earthquakes) and further investigate the effects of structural non-linearity.

Acknowledgements I am earnestly thankful to Prof. Claudio Modena and Prof. Francesca da Porto for creating opportunities for collaboration between the Guangzhou University and the University of Padova. 
Authors' contributions MD: conceptualization, methodology, formal analysis, validation, writing-original draft. EB: formal analysis, investigation. AZ: data curation, visualization. PT: writing-review and editing, supervision. FZ: supervision.

Funding Open access funding provided by Università degli Studi di Padova within the CRUI-CARE Agreement. This research did not receive any specific grant from funding agencies in the public, commercial, or not-for-profit sectors.

Data availability Some or all data and material that support the findings of this study are available from the corresponding author upon reasonable request.

Code availability Calculations were performed in the MATLAB environment.

\section{Declarations}

Conflict of interest The authors declare that they have no conflict of interest.

Open Access This article is licensed under a Creative Commons Attribution 4.0 International License, which permits use, sharing, adaptation, distribution and reproduction in any medium or format, as long as you give appropriate credit to the original author(s) and the source, provide a link to the Creative Commons licence, and indicate if changes were made. The images or other third party material in this article are included in the article's Creative Commons licence, unless indicated otherwise in a credit line to the material. If material is not included in the article's Creative Commons licence and your intended use is not permitted by statutory regulation or exceeds the permitted use, you will need to obtain permission directly from the copyright holder. To view a copy of this licence, visit http://creativecommons.org/licenses/by/4.0/.

\section{References}

Alhan C, Gavin H (2004) A parametric study of linear and non-linear passively damped seismic isolation systems for buildings. Eng Struct 26(4):485-497. https://doi.org/10.1016/j.engstruct.2003.11.004

Altieri D, Tubaldi E, De Angelis M, Patelli E, Dall'Asta A (2018) Reliability-based optimal design of nonlinear viscous dampers for the seismic protection of structural systems. Bull Earthq Eng 16:963-982. https://doi.org/10.1007/s10518-017-0233-4

Ambraseys N, Smit P, Sigbjornsson R, Suhadolc P, Margaris B (2002) Internet-site for European strongmotion data. European Commission, Research-Directorate General

CEN (European Committee for Standardization) (2004) Design of structures for earthquake resistance, part 1: general rules, seismic actions and rules for buildings. Eurocode 8, EN 1998-1. CEN, Brussels

Charmpis DC, Komodromos P, Phocas MC (2012) Optimized earthquake response of multi-storey buildings with seismic isolation at various elevations. Earthq Eng Struct Dyn 41:2289-2310. https://doi.org/10. 1002/eqe. 2187

Charmpis DC, Phocas MC, Komodromos P (2015) Optimized retrofit of multi-storey buildings using seismic isolation at various elevations: assessment for several earthquake excitations. Bull Earthq Eng 13:2745-2768. https://doi.org/10.1007/s10518-015-9737-y

Chen H, Tan P, Zhou F (2017) An improved response spectrum method for non-classically damped systems. Bull Earthq Eng 15:4375-4397. https://doi.org/10.1007/s10518-017-0144-4

Chen H, Hao H, Bi K, Tan P, Peng L, Zhou F (2019) Dynamic analysis of nonclassically damped systems with linear behavior using load-dependent Ritz vectors. Int J Struct Stab Dyn 19(3). https://doi.org/10. $1142 / \mathrm{S} 0219455419500226$

Chey M, Chase J, Mander J, Carr A (2013) Innovative seismic retrofitting strategy of added stories isolation system. Front Struct Civ Eng 7:13-23. https://doi.org/10.1007/s11709-013-0195-9

Coello Coello C, Lamont GB, Van Veldhuizen DA (2007) Evolutionary algorithms for solving multi-objective problems. Genetic and evolutionary computation series., Springer, US

De Domenico D, Ricciardi G, Takewaki I (2019) Design strategies of viscous dampers for seismic protection of building structures: a review. Soil Dyn Earthq Eng 118:144-165. https://doi.org/10.1016/j.soild yn.2018.12.024 
Deb K, Pratap A, Agarwal S, Meyarivan T (2002) A fast and elitist multiobjective genetic algorithm: NSGA-II. IEEE Trans Evol Comput 6(2):182-197. https://doi.org/10.1109/4235.996017

Donà M, Muhr AH, Tecchio G, da Porto F (2017) Experimental characterization, design and modelling of the RBRL seismic-isolation system for lightweight structures. Earthq Eng Struct Dyn 46:831-853. https://doi.org/10.1002/eqe.2833

Donà M, Bernardi E, Zonta A, Minotto M, da Porto F, Tan P (2019) Effectiveness and optimal design of fluid-viscous dampers for inter-storey isolated buildings. In: Proceedings of XVIII ANIDIS conference, Ascoli Piceno, Italia

Dutta A, Sumnicht JF, Mayes RL, Hamburger RO, Citipitioglu A (2009) An innovative application of base isolation technology. In: Proceedings of ATC \& SEI conference on improving the seismic performance of existing buildings and other structures, Francisco, California, pp 841-54

Faiella D, Mele E (2019) Vibration characteristics and higher mode coupling in intermediate isolation systems (IIS): a parametric analysis. Bull Earthq Eng. https://doi.org/10.1007/s10518-019-00637-w

Faiella D, Mele E (2020) Insights into inter-story isolation design through the analysis of two case studies. Eng Struct 215:110660. https://doi.org/10.1016/j.engstruct.2020.110660

Faiella D, Calderoni B, Mele E (2020) Seismic retrofit of existing masonry buildings through inter-story isolation system: a case study and general design criteria. J Earthq Eng. https://doi.org/10.1080/ 13632469.2020.1752854

Fathi M, Makhdoumi A, Parvizi M (2015) Effect of supplemental damping on seismic response of base isolated frames under near \& far field accelerations. KSCE J Civ Eng 19(5):1359-1365. https://doi. org/10.1007/s 12205-014-0101-6

Hall J (1999) Discussion. The role of damping in seismic isolation. Earthq Eng Struct Dyn 28(12):1717-1720

Ismail M, Ikhouane F, Rodellar J (2009) The hysteresis Bouc-Wen model: a survey. Arch Comput Methods Eng 16(02):161-188. https://doi.org/10.1007/s11831-009-9031-8

Kalpakidis IV, Constantinou MC (2008) Effects of heating and load history on the behaviour of leadrubber bearings. Technical report MCEER-08-0027, University at Buffalo, State University of New York, Buffalo, NY

Kelly JM (1999) The role of damping in seismic isolation. Earthq Eng Struct Dyn 28(1):3-20

Koh CG, Balendra T (1989) Seismic response of base isolated buildings including P-delta effects of isolation bearings. Earthq Eng Struct Dyn 18(4):461-473. https://doi.org/10.1002/eqe.4290180402

Liu YH, Wu JB, Donà M (2018) Effectiveness of fluid-viscous dampers for improved seismic performance of inter-storey isolated buildings. Eng Struct 169:276-292. https://doi.org/10.1016/j.engst ruct.2018.05.031

Loh CH, Weng JH, Chen CH, Lu KC (2013) System identification of mid-story isolation building using both ambient and earthquake response data. Struct Control Health Monit 20:139-155

Makris N (1997) Rigidity-plasticity-viscosity: can electro-rheological dampers protect base-isolated structures from near-source ground motions. Earthq Eng Struct Dyn 26:571-91

MIT (Italian Ministry of Infrastructure and Transport) (2018) Update of the "technical standards for constructions". DM 2018/01/17, "S.O. No. 8 alla G.U. del 20 Febbraio 2018, No. 32”, Rome, Italy (in Italian)

Pant DR, Wijeyewickrema AC, ElGawady MA (2013) Appropriate viscous damping for nonlinear time-history analysis of base-isolated reinforced concrete buildings. Earthq Eng Struct Dyn 42(15):2321-2339. https://doi.org/10.1002/eqe.2328

Politopoulos I (2008) A review of adverse effects of damping in seismic isolation. Earthq Eng Struct Dyn 37:447-465. https://doi.org/10.1002/eqe.763

Pollini N, Lavan O, Amir O (2017) Minimum-cost optimization of nonlinear fluid viscous dampers and their supporting members for seismic retrofitting. Earthq Eng Struct Dyn 46:1941-1961. https:// doi.org/10.1002/eqe. 2888

Providakis CP (2008) Effect of LRB isolators and supplemented viscous dampers on seismic isolated buildings under near fault excitations. Eng Struct 32:1187-1198. https://doi.org/10.1016/j.engst ruct.2007.07.020

Ragni L, Micozzi F, Tubaldi E, Dall'Asta A (2020) Behaviour of structures isolated by HDNR bearings at design and service conditions. J Earthq Eng. https://doi.org/10.1080/13632469.2020.1776792

Reggio A, De Angelis M (2015) Optimal energy-based seismic design of non-conventional Tuned Mass Damper (TMD) implemented via inter-story isolation. Earthq Eng Struct Dyn 44:1623-1642. https://doi.org/10.1002/eqe. 2548

Ryan KL, Polanco J (2008) Problems with Rayleigh damping in base-isolated buildings. J Struct Eng. https://doi.org/10.1061/(ASCE)0733-9445(2008)134 
Sues RH, Mau ST, Wen YK (1988) Systems identification of degrading hysteretic restoring forces. J Eng Mech 114(5):833-846

Tan P, Zhang Y, Zhou F (2008) Optimal design and control mechanism study on story isolation system. In: Proceedings of 14th world conference on earthquake engineering, Beijing, China

Tasaka M, Mori N, Yamamoto H, Murakami K, Sueoka T (2008) Applying seismic isolation to buildings in Japan-retrofitting and middle-story isolation. In: Proceedings of 18th analysis and computation specialty conference, ASCE structures congress, Vancouver, BC, Canada

Tubaldi E, Barbato M, Dall'Asta A (2015a) Efficient approach for the reliability-based design of linear damping devices for seismic protection of buildings. ASCE-ASME J Risk Uncertain Eng Syst Part A Civ Eng 2(2):C4015009

Tubaldi E, Ragni L, Dall'Asta A (2015b) Probabilistic seismic response assessment of linear systems equipped with nonlinear viscous dampers. Earthq Eng Struct Dyn 44(1):101-120. https://doi.org/10. $1002 /$ eqe. 2461

Wang SJ, Chang KC, Hwang JS, Lee BH (2011) Simplified analysis of mid-storey seismically isolated buildings. Earthq Eng Struct Dyn 40:119-133. https://doi.org/10.1002/eqe.1004

Wolff ED, Ipek C, Constantinou MC, Tapan M (2015) Effect of viscous damping devices on the response of seismically isolated structures. Earthq Eng Struct Dyn 44:185-198. https://doi.org/10.1002/eqe.2464

Xie Y, Zhang J (2017) Optimal design of seismic protective devices for highway bridges using performancebased methodology and multiobjective genetic optimization. J Bridge Eng 22(3):04016129. https://doi. org/10.1061/(ASCE)BE.1943-5592.0001009

Zhang R, Phillips BM, Taniguchi S, Ikenaga M, Ikago K (2017) Shake table real-time hybrid simulation techniques for the performance evaluation of buildings with inter-story isolation. Struct Control Health. https://doi.org/10.1002/stc.1971

Zhou FL (2001) Seismic isolation of civil buildings in the People's Republic of China. Progr Struct Eng Mater 3(3):268-276. https://doi.org/10.1002/pse.85

Zhou FL, Yang Z, Liu WG, Tan P (2004) New seismic isolation system for irregular structure with the largest isolation building area in the world. In: Proceedings of 13th world conference on earthquake engineering, Vancouver, BC, Canada

Zhou Q, Singh MP, Huang XY (2016) Model reduction and optimal parameters of mid-story isolation systems. Eng Struct 124:36-48. https://doi.org/10.1016/j.engstruct.2016.06.011

Ziyaeifar M, Noguchi T (1998) Partial mass isolation in tall buildings. Earthq Eng Struct Dyn 27(199801):49-65

Publisher's Note Springer Nature remains neutral with regard to jurisdictional claims in published maps and institutional affiliations. 